\title{
Efficacités comparées des processus naturels d'épuration des eaux de surface: unités fonctionnelles lotiques, connectées au Rhin (système île de Rhinau, France)
}

Comparison between the different self purifying process in surface waters: running waters systems connected with the River Rhine (Rhinau Island, France)

\author{
Robach Fabienne, Eglin Isabelle, Trémolières Michèle \\ Université Louis Pasteur. CEREG. 74 route du Rhin. B.P. 10. F 67048 Strasbourg Cedex
}

Résumé. - Les capacités naturelles d'amélioration de la qualité de l'eau (réduction de la pollution organique et réduction de la charge eutrophisante) de différentes unités fonctionnelles lotiques de l'hydrosystème rhénan dans le secteur inondable de l'île de Rhinau ont été estimées et comparées, en fonction du degré d'artificialisation des cours d'eau et de la composition de la végétation aquatique.

Les paramètres discriminants de la qualité de l'eau retenus sont les suivants: concentrations en $\mathrm{NH}_{4}, \mathrm{PO}_{4}^{-}, \mathrm{NO}_{3}^{-}$en ce qui concerne la charge eutrophisante, $\mathrm{DBO}_{5}, \mathrm{DCO}$, comptages bactériens en ce qui concerne la charge en matière organique.

Le cours d'eau le plus artificialisé (Rhin canalisé), quasiment dépourvu de toute végétation aquatique supérieure, au tracé rectiligne, aux berges bétonnées et aux eaux turbides, se comporte comme un système inerte: il se révèle incapable (sur $23,5 \mathrm{~km}$ ) d'amortir ou de réduire les charges polluantes et eutrophisantes. Inversement, les cours d'eau forestiers de tresse, qui sont fortement végétalisés et qui se rapprochent le plus d'un "état naturel ", présentent une remarquable capacité épuratrice, exaltée en période estivale.

L'efficacité des processus d'amélioration de la qualité de l'eau est optimisée en période végétative dans les systèmes végétalisés, ce qui confirme le rôle prédominant des biocénoses aquatiques. Les portions de cours d'eau non végétalisées mais dont le lit est limono-vaseux, présentent quant à elles une capacité épuratrice moindre, en raison des processus physico-chimiques d'absorption ou de précipitation dans le sédiment.

Mots-clés. - Eutrophisation, Auto-épuration, Phosphore, Azote, Rhin, Macrophytes aquatiques

Summary. - The self purification capacity (organic matter pollution $\left(\mathrm{BOD}_{5}\right)$ and eutrophication ( $\mathrm{PO}_{4}^{-}, \mathrm{NH}_{4}^{+}, \mathrm{NO}_{3}$ ), of some running waters systems has been estimated in an rhenan flooded area. Rhinau Island (Alsace, France) has been chosen because of its particular status as a flooded sector in the Upper Rhine alluvial valley. 
These capacities were compared, according with the artificialisation level and the composition of the aquatic phytocoenoses. A completely artificial stream (canalised Rhine) or partly artificial stream (straightened Rhine: "Old-Rhine") is also compared to more natural systems, more or less connected to the Rhine wich flows in an alluvial forest.

It is concluded by the results, that the improvement of water quality from upstream to downstream in the running waters is accentuated in the less artificial systems, and during the growing season thanks to vegetation action. This fact exhibits the major role of the aquatic vegetation in the self purifying process.

By contrast, the canalised Rhine, is an inert system. The water quality does not change over a distance of $23.5 \mathrm{~km}$. The parts of the water bodies which are not vegetated, exhibit lower self purifying capacities than the vegetated area. In this case, the low decrease of phosphore can be explained by physico-chemical processes (adsorption, precipitation in the sediment).

\section{PROBLÉMATIQUE}

Si l'existence des processus naturels d'amélioration de la qualité de l'eau est reconnue depuis longtemps (la notion de zones de saprobies, qui reconnait implicitement le processus de réduction de la matière organique, date du début du siècle: elle est due à Kolkwits et Marsson (1909)), le déterminisme des mécanismes biologiques et (ou) abiotiques qui régulent cette épuration naturelle fait encore l'objet de nombreuses controverses scientifiques (Blake 1989, Radoux 1988...).

On distingue classiquement deux processus interactifs d'amélioration de la qualité de l'eau, qui se succèdent ou se complètent dans le cas d'une pollution organique localisée, mais qui agissent conjointement dans le cas d'une pollution-eutrophisation (que nous désignerons sous le terme de pollution couplée) diffuse:

- la réduction de la charge en matières organiques fermentescibles, processus habituellement désigné sous le nom d' "auto-épuration»: il semble admis que cette première étape dans l'épuration des eaux soit essentiellement déterminée par l'action biologique de micro-organismes saprophytes. II s'agit donc d'une activité fermentaire, elle même influencée par des facteurs physiques tels que la vitesse du courant, les débits respectifs de la charge polluante et des eaux réceptrices, la température... Cette étape se traduit par une réduction par minéralisation de la matière organique dégradable, ce qui induit nécessairement une augmentation de la charge en éléments minéraux eutrophisants: ammoniaque $\left(1^{\text {re }}\right.$ étape de la décomposition de la matière organique), et phosphates notamment.

- la réduction de la charge eutrophisante: Cette étape succède généralement aux processus de réduction de la charge en matières organiques. Mais elle peut également agir de concert, dans le cas d'une pollution couplée. Les modalités qui intervien- 
nent au cours de cette étape (phénomènes de sorption-désorption, ou phénomènes biologiques d'absorption et de fixation dans la biomasse) sont complexes et leur importance relative est encore mal connue.

Les processus d'amélioration de la qualité de l'eau qui concernent l'ensemble des éléments fertilisants (phosphore et azote essentiellement), ont été particulièrement étudiés dans le cas du phosphore (orthophosphates solubles) dont on sait qu'il est l'élément clé des phénomènes d'eutrophisation (Arvieu 1980, Carbiener 1990, Carpenter et Adams 1977, Chen et Barko 1988, Kramer et al., 1972, Ombredanne et al., 1982...).

Le cycle du phosphore est soumis à des processus chimiques (précipitation-solubilisation, adsorption-désorption), et à des processus biologiques par absorption et fixation dans la biomasse (sous forme d'orthophosphates solubles)-excrétion-dégradation de la matière organique. Les processus de réduction de la charge en phosphore dans un système aquatique ne sont pas, à l'échelle de l'écosystème, des processus d'élimination du phosphore. II s'agit en fait, de mécanismes de transfert d'un compartiment à l'autre (sédiment-eauplante), ou d'exportation vers l'aval, la charge totale potentielle en phosphore ne diminuant pas réellement. La biodisponibilité du phosphore sera fonction de ces mécanismes de transfert et d'échange.

Notons enfin que la chimie du phosphore dans les sédiments est fré- quemment dominée par les interactions entre les différentes espèces de fer (les phosphates ferriques étant insolubles, tandis que les phosphates ferreux sont solubles).

Les principaux paramètres et mécanismes qui déterminent l'adsorption et la désorption du phosphore à partir du sédiment sont: le $\mathrm{pH}$, les teneurs en Calcium, le degré d'agitation du sédiment et le potentiel oxydo-réducteur.

Si le rôle des végétaux aquatiques dans le cycle biogéochimique de certains nutriments a été étudié dans plusieurs travaux (Blake 1989, Bole et Allan 1978, Fogg 1973, Radoux et Kemp 1988), l'effet épurateur des macrophytes aquatiques, vis-à-vis de ces mêmes éléments, n'a été que rarement quantifié. Quelques valeurs de rétention journalières ont néanmoins été relevées: de $0,11 \mathrm{~g} \mathrm{P. \textrm {m } ^ { - 2 }}$ pour Salvinia (espèce de fougère aquatique) à $0,22 \mathrm{~g} \mathrm{P} . \mathrm{m}^{-2}$ pour Ceratophyllum (Blake 1989).

Le cycle de l'azote, quand à lui, est essentiellement dépendant des processus microbiologiques dans l'eau et le sédiment (Pilleboue-Baptendier, 1987), même si, mais dans une moindre importance, se déroulent dans la phase sédiment et sur les particules en suspension, des processus de sorption-désorption d'azote ammoniacal et organique (Ombredanne et al., 1982).

Dans la suite de cet exposé, nous réserverons le terme "auto-épuration" à la réduction de la charge en matière organique. Le processus 
global d'amélioration de la qualité de l'eau dépendra donc de l'activité auto-épuratrice d'une part, des processus de réduction de la charge euphorisante d'autre part.

Bénéficiant avec l'île de Rhinau, d'un laboratoire de recherche naturel exceptionnel par sa richesse et par sa complexité (mais néanmoins affecté par la canalisation du Rhin), nous disposons d'une "miniaturisation naturelle" de l'ensemble alluvial rhénan du secteur des tresses et anastomoses. Ce modèle réduit présente, en effet, les principales unités fonctionnelles aquatiques, lotiques et lénitiques, qu'un ensemble alluvial peut receler. Nous nous sommes attachés plus particulièrement à l'étude et à la quantification des capacités d'amélioration de la qualité de l'eau (autoépuration et réduction de la charge eutrophisante) des différentes unités fonctionnelles lotiques, en fonction du degré d'artificialisation (présence-absence de végétation, composition de la végétation aquatique, présence-absence de sédiment...).

\section{SITE ET MÉTHODE}

\subsection{Site}

Au sein de I'hydrosystème fluvial rhénan nous avons retenu pour dition le secteur de Rhinau-Gerstheim, situé à une trentaine de $\mathrm{km}$ au Sud de Strasbourg, dans l'ancienne zone dite des "tresses et anastomoses" (fig. 1). Ce secteur est encore représentatif du fonctionnement naturel de l'hydrosystème rhénan, quoique à une échelle très restreinte. II présente une grande diversité de types de milieux aquatiques: milieux lotiques ou lénitiques, connectés ou déconnectés du fleuve, ils entretiennent tous avec le Rhin des relations plus ou moins étroites (par l'intermédiaire de la nappe phréatique influencée par des infiltrations rhénanes ou par le biais des inondations).

Dans ce secteur on distingue trois types de systèmes lotiques: le Rhin canalisé, les Vieux-Rhin, les bras de tresse (fig. 2).

- Le Rhin canalisé, avec ses rives bétonnées et surélevées est un milieu artificialisé, quasi dépourvu de macrophytes aquatiques, qui domine, de plus d'une dizaine de metres par endroit, sa plaine alluviale. Malgré ce carcan de béton, les fuites du Rhin dans son lit sont loin d'être négligeables et ont été évaluées à $3 \mathrm{~m}^{3} \cdot \mathrm{s}^{-1}$ (au cours des années 70) entre Marckolsheim et Strasbourg, et à $0,6 \mathrm{~m}^{3} \cdot \mathrm{s}^{-1}$ au niveau du " coude de Rhinau " (BRGM-SGAL 1978). La nappe phréatique de la trange riveraine du fleuve est ainsi fortement contaminée par ces infiltrations qui se traduisent par de fortes teneurs en phosphates et en chlorures notamment, et par de faibles concentrations en nitrates comme dans l'ensemble du bassin du Rhin supérieur, et contrairement à la partie ellane (nappe de l'III) de la nappe phréatique alsacienne. Au niveau de chacun des biefs une dérivation longe les îles à l'Ouest et amène une partie des eaux du fleuve 

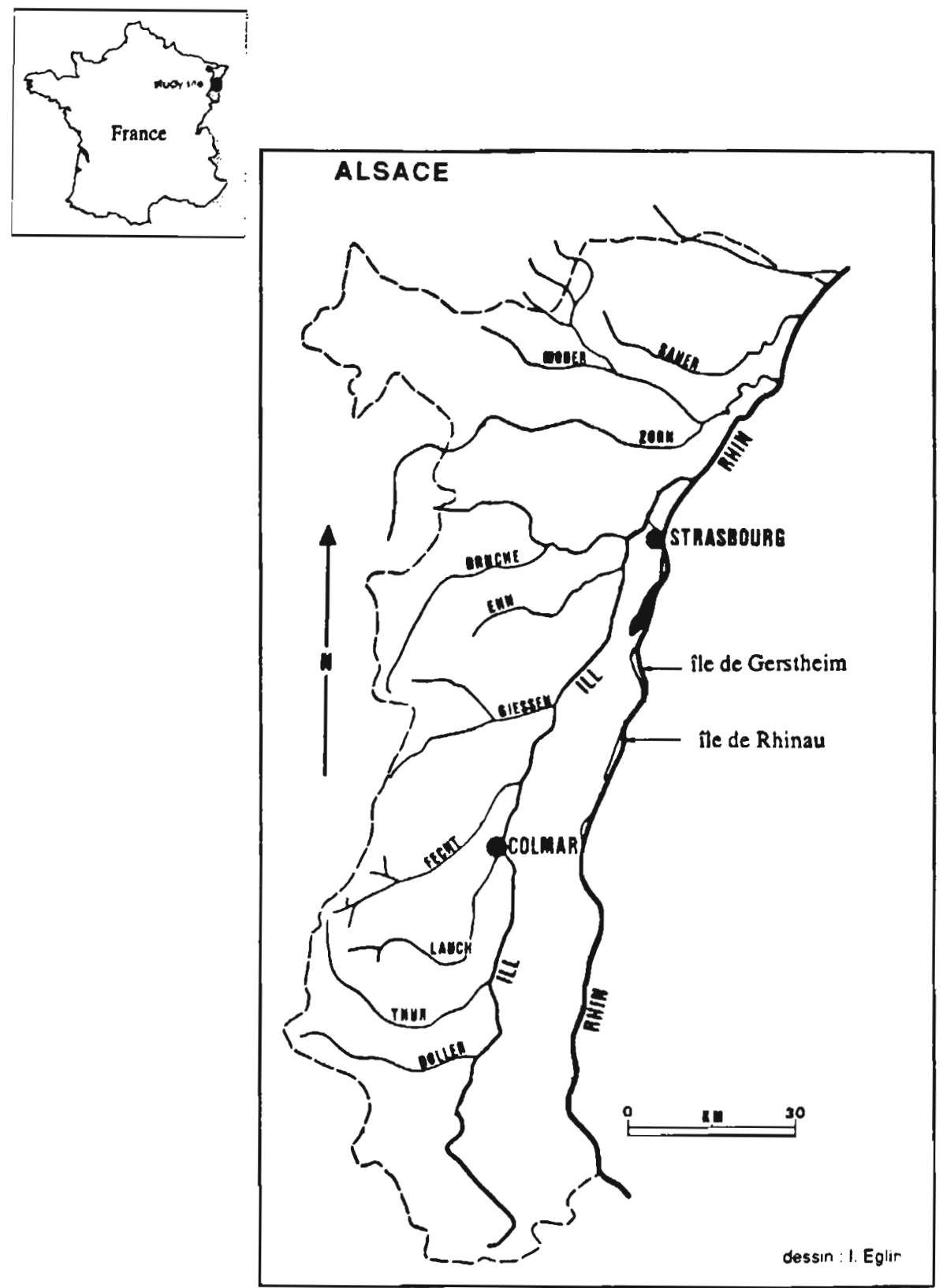

Fig. 1. - Zone d'étude (plaine d'Alsace, France).

Fig. 1. - Sludy area (Alsacian plain, France). 


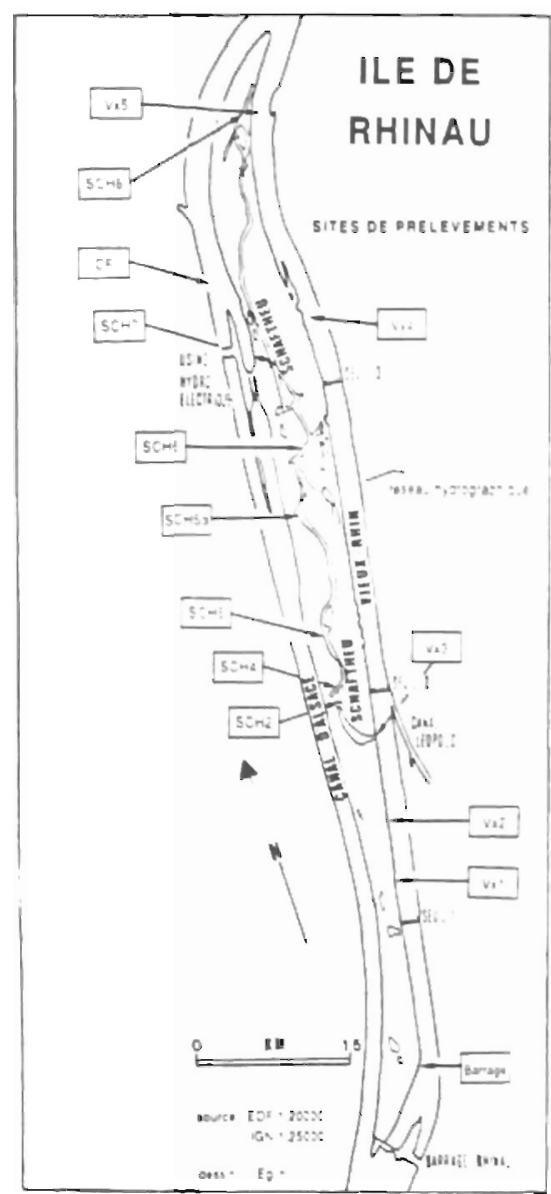

Fig. 2. - Sites de prélévements.

Fig. 2. - Sampling sites.

canalisé vers une usine hydroélectrique.

Nous avons échantillonné 5 points sur le Rhin canalisé, d'amont en avál:

- Plan incliné de Schœnau (PI1)

- Canal de fuite de l'usine hydroélectrique de Rhinau (CF)

- Bac de Rhinau (BAC)

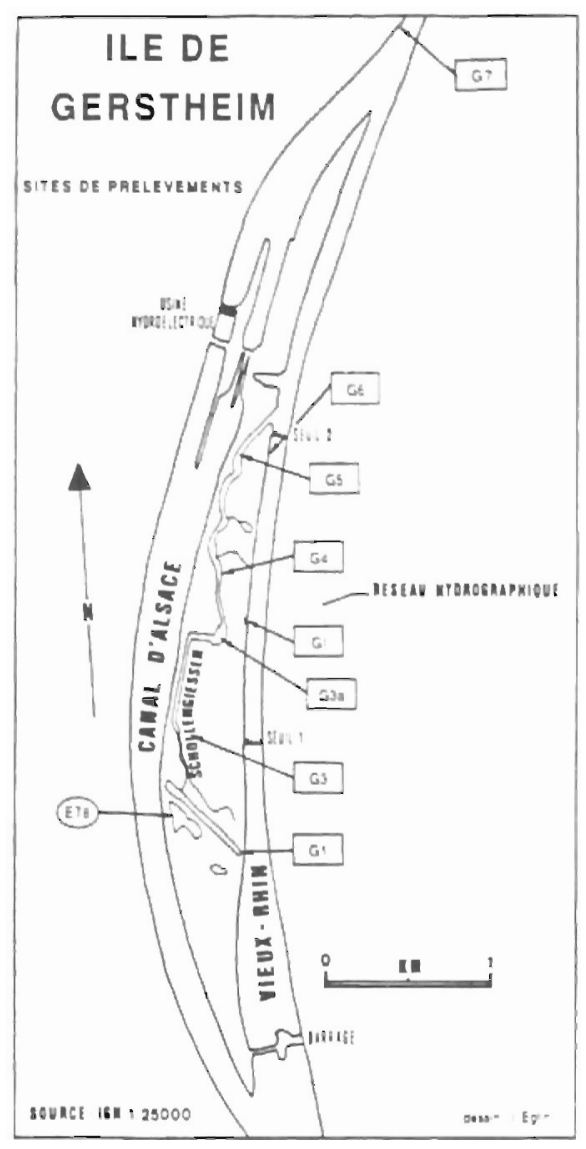

- Plan incliné entre Rhinau et Gerstheim (PI2)

- Canal de fuite de l'usine hydroélectrique de Gerstheim (G7)

- Le Vieux-Rhin correspond au Rhin rectifié du $19^{e}$ siècle. II est alimenté directement par le Rhin canalisé au niveau d'un barrage situé en amont de chaque île. Au niveau du 
bief de Rhinau, le débit réservé est de $15 \mathrm{~m}^{3} \cdot \mathrm{s}^{-1}$, mais dès que le débit du fleuve canalisé dépasse la capacité des turbines de l'usine hydroélectrique EDF, une partie des eaux est déviée vers l'ancien cours du fleuve. Lorsque ce débit atteint 1700 à $2000 \mathrm{~m}^{3} \cdot \mathrm{s}^{-1}$, le Vieux-Rhin déborde et les eaux de crue viennent s'épancher au sein de la luxuriante végétation de la forêt alluviale des îles. Le cours du Vieux-Rhin est jalonné de seuils servant à soutenir le niveau de la nappe phréatique.

Au niveau de l'îe de Rhinau le Vieux-Rhin est malheureusement perturbé par des apports d'eau hyper-eutrophisants en provenance d'un petit canal allemand (canal Leopold) drainant toute la zone de Fribourg et passant par une unique station d'épuration, dépourvue de système d'épuration tertiaire. Ce petit affluent vient se jeter dans le Vieux-Rhin quelques dizaines de mètres en amont du seuil 2.

Sur le Vieux-Rhin de Rhinau nous disposons des points suivants (Barrage, $V \times 1, V \times 2, V \times 3, V \times 4, V \times 5)$, et à Gerstheim (G1, GI, G6).

\section{- Les anciens cours de tresse}

- Le Schaftheu, ancien bras latéral de tresse, parcours l'île de Rhinau en traversant la forêt alluviale rhénane. Il est alimenté à l'amont par une prise d'eau sur le Vieux-Rhin et retourne à ce dernier à l'aval de l'île après un parcours de $7,5 \mathrm{~km}$ environ, laissant alterner les seuils et les tronçons plus profonds à courant lent. Dans sa partie amont, le Schaftheu bénéficie d'apports d'eaux phréatiques (suintements phréatiques à travers le fond du lit du cours d'eau...), le toit de la nappe étant plus élevé dans ce secteur que le fond du lit. Dans la partie aval de cet ancien cours de tresse, par contre, l'inverse se produit, et cette fois c'est la nappe qui draine le Schaftheu (Sanchez Perrez et al., 1991).

Malheureusement, comme précédemment pour le Vieux-Rhin, les eaux peuvent être fortement contaminées par les apports hyper-eutrophes du Canal Leopold, et ceci en deux points: tout d'abord au niveau de la prise d'eau elle-même qui est située à quelques dizaines de mètres en amont et vis-à-vis de la confluence du canal allemand avec le Vieux-Rhin (prise d'eau qui peut être atteinte par les remous eutrophes dans le cas d'épisodes de vent du Nord notamment), et plus en aval, au niveau d'un point de contact entre le Vieux-Rhin contaminé et le Schaftheu, juste en amont du seuil 3 (point SCH6 sur le Schaftheu).

Sur le Schaftheu (fig. 2), nous avons, d'amont en aval les points: Vx3 (prise d'eau), SCH2, SCH4 (diverticule aveugle à alimentation phréatique), SCH5 (seuil), SCH5a, SCH6, SCH7, SCH8 (quasiment à la confluence avec le Vieux-Rhin).

- Le Schollengiessen de l'íle de Gersheim (fig. 2), long d'environ $3,8 \mathrm{~km}$, présente un cours très uniforme (lame d'eau peu importante dans l'ensemble, fond quasi uniformément graveleux) et la végétation aquatique $y$ est moins bien dévelop- 
pée qu'à Rhinau, à l'exception d'une profonde fosse (à alimentation phréatique) située à peu près à la moitié du parcours.

Nous disposons de 5 points sur le Schollengiessen: G1 (prise d'eau), G3, G3a (aval de la fosse), G4, G5 (amont de la confluence avec le Vieux-Rhin).

Nous avons étudié mensuellement l'ensemble de ces points depuis mars 1988 pour l'île de Rhinau (Eglin et Robach 1992, Robach et Eglin 1988, Robach et al., 1990), Novembre 1989 pour l'île de Gerstheim. Les principales caractéristiques du niveau trophique de ces unités figurent dans le tableau I.

\subsection{Méthodes}

Les prélèvements d'eau ont été effectués pour chaque site selon un pas de temps mensuel. Ces prélèvements se sont toujours réalisés selon une progression d'amont en aval. En période estivale, des relevés de la végétation aquatique ont été effectués parallèlement.

La Température (OXI 96 WTW), le $\mathrm{pH}$ (HI 8424 Labo-Moderne), I'O2 dissous et le taux de saturation en $\mathrm{O} 2$ (OXI 96 WTW) ont été mesuré in situ.

Dans le cas du suivi de nos différentes unités fonctionnelles, la mesure de la température permet notamment de mettre en évidence l'existence d'apports d'eaux souterraines dans le système. En effet, les eaux phréatiques sont des eaux sténothermes froides (les températures varient entre 10 et $13^{\circ} \mathrm{C}$ ). Le taux de saturation en $\mathrm{O}_{2}$, quant à lui, permet d'une part d'estimer la capacité d'une eau de surface à s'auto-épurer, et d'autre part de révéler la présence

Tableau I. - Teneurs moyennes en $\mathrm{NH}_{4}^{+}, \mathrm{PO}_{4}^{3-}, \mathrm{NO}_{3}^{-}$et $\mathrm{DBO}_{5}$ des principales unités fonctionnelles lotiques des iles de Rhinau et Gerstheim.

Table I. $-\mathrm{NH}_{4}^{+}, \mathrm{PO}_{4}^{3-}, \mathrm{NO}_{3}^{-}$average content and $\mathrm{DBO}_{5}$ values of the main running waters systems in Rhinau Island and Gerstheim Island.

\begin{tabular}{|c|c|c|c|c|c|c|}
\hline \multirow{2}{*}{$\begin{array}{l}\text { Paramè- } \\
\text { tres }\end{array}$} & \multirow[t]{2}{*}{ Période } & \multirow[t]{2}{*}{ Rhin } & \multirow{2}{*}{$\begin{array}{l}\text { Vieux- } \\
\text { Rhin }\end{array}$} & \multirow{2}{*}{$\begin{array}{l}\text { Schollen- } \\
\text { giessen }\end{array}$} & \multicolumn{2}{|c|}{ Schaftheu } \\
\hline & & & & & $\begin{array}{l}\text { portion non } \\
\text { végétalisée }\end{array}$ & $\begin{array}{l}\text { portion } \\
\text { végétalisée }\end{array}$ \\
\hline $\begin{array}{r}\mathrm{N}-\mathrm{NH} 4+ \\
(\mu \mathrm{g} / \mathrm{l})\end{array}$ & $\begin{array}{l}\text { Moy. période vég. } \\
\text { Moy. hors période vég. }\end{array}$ & $\begin{array}{l}123 \\
246\end{array}$ & $\begin{array}{r}98 \\
208\end{array}$ & $\begin{array}{l}101 \\
234\end{array}$ & $\begin{array}{l}222 \\
278\end{array}$ & $\begin{array}{l}332 \\
183\end{array}$ \\
\hline $\begin{array}{l}\text { P-PO43- } \\
(\mu \mathrm{g} / \mathrm{l})\end{array}$ & $\begin{array}{l}\text { Moy. période vég. } \\
\text { Moy. hors période vég. }\end{array}$ & $\begin{array}{l}45 \\
56\end{array}$ & $\begin{array}{l}36 \\
51\end{array}$ & $\begin{array}{l}36 \\
77\end{array}$ & $\begin{array}{r}102 \\
75\end{array}$ & $\begin{array}{r}120 \\
52\end{array}$ \\
\hline $\begin{array}{l}\mathrm{N}-\mathrm{NO} 3- \\
(\mathrm{mg} / \mathrm{l})\end{array}$ & $\begin{array}{l}\text { Moy. période vég. } \\
\text { Moy. hors période vég. }\end{array}$ & $\begin{array}{l}1,14 \\
1,77\end{array}$ & $\begin{array}{l}1,15 \\
1,58\end{array}$ & $\begin{array}{l}1,13 \\
1,76\end{array}$ & $\begin{array}{l}1,26 \\
1,52\end{array}$ & $\begin{array}{l}0,98 \\
1,36\end{array}$ \\
\hline $\begin{array}{l}\mathrm{DBO5} \\
\text { (mgO2/l) }\end{array}$ & $\begin{array}{l}\text { Moy. période vég. } \\
\text { Moy. hors période vég. }\end{array}$ & $\begin{array}{l}2,5 \\
3,3\end{array}$ & $\begin{array}{l}2,92 \\
1,95\end{array}$ & $\begin{array}{l}3,27 \\
2,04\end{array}$ & $\begin{array}{l}4,68 \\
3,14\end{array}$ & $\begin{array}{l}4,2 \\
2,89\end{array}$ \\
\hline
\end{tabular}


d'une eau d'origine phréatique. En effet, celle-ci, pauvre en éléments nutritifs et de faible productivité, est naturellement pauvre en oxygène, de l'ordre de 35 à $40 \%$ pour les résurgences phréatiques du ried de l'Ill (Carbiener 1983).

Afin de déterminer, les niveaux de trophie et de saprobie des différents sites étudiés d'une part, l'évolution spatio-temporelle de l'auto-épuration et de la réduction de la charge eutrophisante d'autre part, les paramètres discriminants de la qualité de l'eau suivants ont été étudiés: orthophosphates, $\mathrm{N}$-ammoniacal, $\mathrm{N}$-nitrique...). Cette étude a été complétée par l'utilisation de traceurs hydrologiques (notamment les ions $\mathrm{Cl}^{-}$). Ce choix est basé sur plusieurs études antérieures (Carbiener et Ortscheit 1987, Carbiener et al., 1988, Carbiener et al., 1990).

\subsubsection{Détermination de la charge eutrophisante: paramètres discri- minants}

Dans les milieux aquatiques de la plaine rhénane alsacienne, les paramètres suivants ont été déterminés comme étant discriminants pour la qualité de l'eau: orthophosphates, $\mathrm{N}$ nitrique, $\mathrm{N}$-ammoniacal (Carbiener et Ortscheit 1987, Carbiener et al. 1990).

Les orthophosphates et l'azote ammoniacal ont été dosés par des méthodes spectrophotométriques (AFNOR (1986): NF T 90-023 et NF T 90-015 adaptées à l'utilisation d'un auto-analyseur à microflux continu. L'azote nitrique a été dosé par colorimétrie (formation d'un composé sulfoné nitré, en présence d'acide sulfurique et de salicylate de sodium. Ce composé produit une coloration jaune stable avec le tartrate double de sodium et potassium).

\subsubsection{Evaluation de la pollution organique}

- Demande Chimique en $\mathrm{O} 2$ (DCO) : AFNOR (1986) NF 90-101

- Demande Biochimique en $\mathrm{O} 2$ sur 5 jours (DBO5)

- Comptages bactériens

Méthode utilisée: Dénombrement bactérien après mise en culture d'une aliquot d'eau à analyser, sur gélose (enrichie en sang de mouton). Passage à l'étuve à $30^{\circ} \mathrm{C}$, puis comptage après $24 \mathrm{~h}$ et $48 \mathrm{~h}$.

- Dosage de la chlorophylle a: AFNOR (1986) T 90-117

- Dosage de l'azote et du phosphore total (sédiment et plante):

Dosage de l'Azote total après minéralisation (méthode de Kjeldahl) et dosage du Phosphore total par la digestion à l'acide perchlorique (AFNOR NT 7 90-023 in Didenot et al., 1987). Ces mêmes méthodes ont été appliquées pour doser l'Azote et le Phosphore total contenus dans les dépôts calcaires précipités sur la face supérieure des feuilles de macrophytes aquatiques submergés.

- Evaluation du pourcentage de matières organiques dans le sédiment: le taux de matière organique fine a été estimé à l'aide de la méthode de perte au feu telle qu'elle a été décrite dans Sanchez-Perez 
(1987) d'après les études réalisées à ce sujet par divers auteurs comme Ball (1964) et Davis (1974), in Sanchez-Perez (1987).

\section{RÉSULTATS}

Les capacités respectives d'épuration (de la charge en matière organique et eutrophisante) de chacune des unités fonctionnelles de l'île de Rhinau ont été testées. Ce processus naturel d'amélioration de la qualité de l'eau s'inscrit essentiellement dans une dimension spatiale (épuration au cours du transfert amont-aval).

Nous avons estimé l'efficacité d'un écosystème aquatique d'eau courante, à soustraire de la phase aqueuse et au cours du transfert amont-aval, un élément $X_{1}$ par la formule $(A)$

$$
(A): E(x)=\frac{(x 2-x 1)}{x 1 \cdot d}
$$

où

$E(x)$ représente la capacité épuratrice vis-à-vis de l'élément $X$, au cours du transfert amont-aval,

$x 1$ représente la teneur de l'élément $X$ au point de référence amont en $\mu \mathrm{g} \cdot \mathrm{l}^{-1}$,

x2 la teneur de l'élément $X$ au point de référence situé en aval en $\mu \mathrm{g} \cdot \mathrm{l}^{-1}$. d représente la distance séparant les points $\times 1$ et $\times 2$ (en $\mathrm{km}$ ).

Les débits respectifs, et donc le volume d'eau concerné, de chaque unité lotique restant quasi constants au cours de l'année dans notre secteur d'étude (excepté en période de hautes eaux) on peut raisonnablement comparer, d'une campagne de prélèvement à l'autre, l'évolution "amont-aval" de chacun des paramètres mesurés.

Les principaux résultats concernent l'évolution amont-aval des paramètres les plus discriminants de la trophie $\left(\mathrm{P}-\mathrm{PO}_{4}^{3}, \mathrm{~N}-\mathrm{NH}_{4}^{+}\right)$et de la saprobie $\left(\mathrm{DBO}_{5}, \mathrm{DCO}\right)$. Pour chaque année de prélèvement (de 1989 à 1990), les moyennes annuelles, les moyennes des périodes végétatives (de mai à septembre) et les moyennes des périodes non végétatives ont été calculées, et seront utilisées dans la détermination des aptitudes épuratrices respectives des unités fonctionnelles (tableau I).

Nous utilisons également pour l'ensemble des trois années de prélèvement, les moyennes annuelles (toutes années confondues), les moyennes de périodes végétatives et les moyennes de périodes non végétatives (sur 3 ans).

Afin de vérifier si les moyennes "amont" et les moyennes "aval" étaient significativement différentes, elles furent comparées deux à deux, par la méthode des couples (échantillons dépendants, logiciel Statitcf).

\subsection{Le Rhin canalisé}

\subsubsection{Evolution amont-aval}

Le Rhin canalisé, se définit comme un système écologique ouvert et haute- 
ment artificialisé. II se comporte visà-vis de la réduction des charges eutrophisantes et de l'activité autoépuratrice, comme un système de transmission quasi inerte. En effet, les teneurs en phosphates, azote ammoniacal, DCO et DBO5 (tableaux II et III) n'évoluent pas de manière significative (au seuil de $5 \%$ ) au cours du transfert amont-aval, entre les deux points de référence ( $\mathrm{PI}$ et $\mathrm{BAC})$ distants de $23,5 \mathrm{~km}$ environ. En effet, nous n'observons pas, pour l'ensemble des paramètres pré-cités de diminutions significatives (au seuil de $5 \%$ ) entre les moyennes annuelles du point de référence amont (PI) et les moyennes annuelles du point de référence situé en aval (BAC). L'année 1989, année particulièrement sèche, présente même une légère augmentation des teneurs moyennes en azote ammoniacal.

\subsubsection{Evolution saisonnière}

Nous constatons, de la même manière, que les moyennes des teneurs (en phosphates notamment) mesurées pendant les périodes végétatives (de mai à septembre), ne diffèrent pas significativement des valeurs mesurées hors des périodes végétatives (tableau II). Cette absence de différenciation saisonnière est à rapprocher de la quasi absence de toute flore macrophytique développée. En effet, seules quelques plantes eurytopes (dont Potamogeton pectinatus) survivent, se présentant alors sous la forme de quelques plages de dimension réduite, lorsque des enrochements localisés permettent l'ancrage de leur système racinaire. Les aspérités des berges bétonnées permettent, quant à elles, l'installation d'une flore algale composées essentiellement de Cladophora sp. Hormis ces maigres populations végétales, qui s'installent généralement à proximité des berges et à faible profondeur, les eaux turbides inhibent toute autre activité photosynthétique en profondeur.

\subsubsection{Capacité épuratrice globale}

Les capacités épuratrices du fleuve canalisé sont caractérisées par des irrégularités lors du transfert amontaval, vis-à-vis des charges eutrophisantes et polluantes et semblent sans relation avec des périodicités saisonnières. On distingue même des périodes où les capacités épuratrices sont négatives, ce qui correspond à des augmentations des teneurs mesurées (fig. 3). Ces anomalies (le fleuve ne reçoit aucun affluent en rive gauche entre les deux points $\mathrm{PI}$ et BAC) pourraient être imputées pour partie, aux apports hypertrophes du canal Leopold qui se déversent indirectement dans le Rhin par l'intermédiaire du Vieux-Rhin. Mais en raison du débit relativement faible du canal allemand (inférieur à $50 \mathrm{~m}^{3} \cdot \mathrm{s}^{-1}$ alors que celui du Rhin dépasse généralement $400 \mathrm{~m}^{3} \cdot \mathrm{s}^{-1}$ même en période d'étiage), il semble plus probable que la majeure partie des augmentations de charges eutrophisantes provienne soit de phénomènes physiques de sorption-désorption à partir du sédiment (épaisses couches de sédiments limono-vaseux bloqués en amont des barrages) ou à partir des particules en 
Tableau II. - Teneurs moyennes et fluctuations saisonnières en $\mathrm{NH}_{4}^{+}$et $\mathrm{PO}_{4}^{3-}$ du Rhin canalisé aux points PI et BAC (distant de $23,5 \mathrm{~km}$ ).

Table II. $-\mathrm{NH}_{4}^{r}$ and $\mathrm{PO}_{4}^{3}$ average contents and seasonal variations of the canalised Rhine.

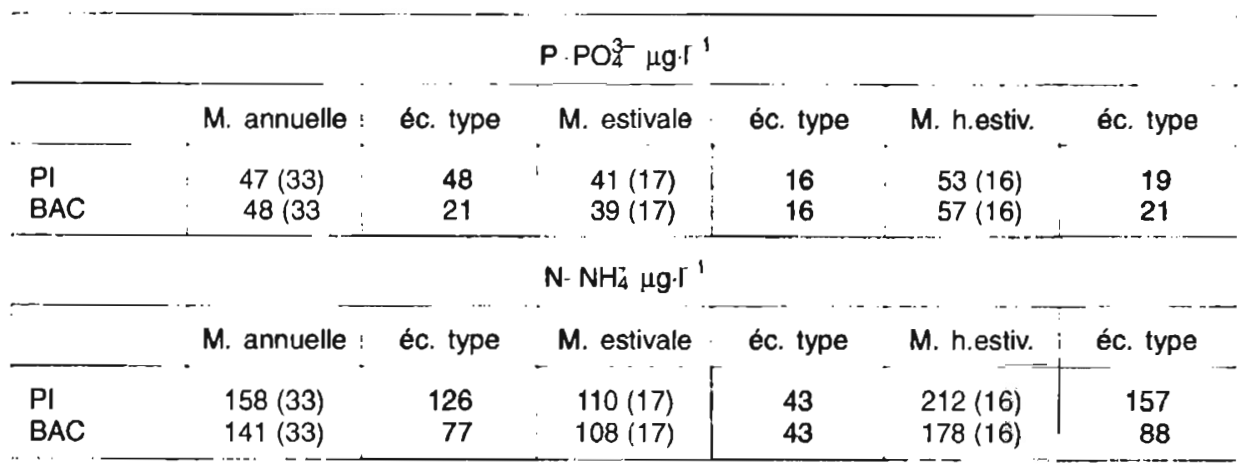

Tableau III. - Valeurs moyennes de la $\mathrm{DBO}_{5}$, en période végétative (mai à septembre) dans le Rhin canalisé.

Table III. - $\mathrm{BOD}_{5}$ mean values, during vegetative period (may to september) in the canalised Rhine.

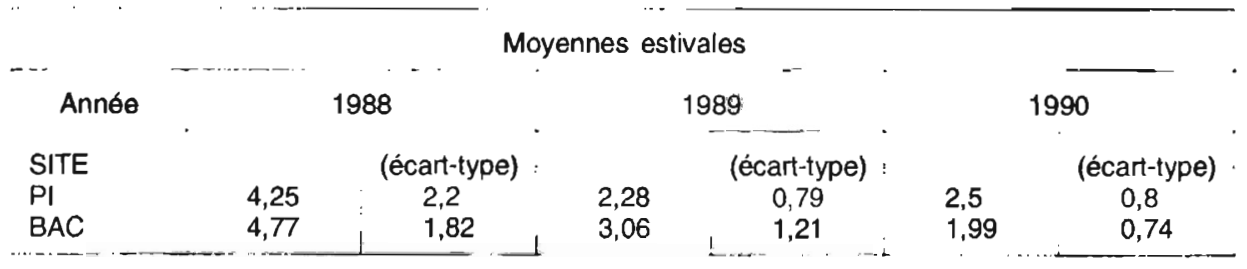

suspension (MST de Gerstheim pouvant dépasser les $50 \mathrm{mg} \cdot \mathrm{I}^{-1}$ dans le Vieux-Rhin de Gerstheim), soit de “rejets sauvages " d'eaux usées.

Au cours de ces trois années d'étude, le Rhin canalisé s'est caractérisé

- d'une part: par une incapacité globale à réduire les charges eutrophisantes et polluantes sur une distance de $23,5 \mathrm{~km}$,
- d'autre part: par un comportement très irrégulier vis-à-vis de ces charges, faisant alterner des périodes de très faible activité épuratrices à des périodes d'augmentation des charges. Ces dernières semblent essentiellement dépendantes de phénomènes météorologiques (l'augmentation des teneurs en phosphates et en azote ammoniacal en juillet 1989 est à corréler avec un mois particulièrement chaud et orageux, ce qui 

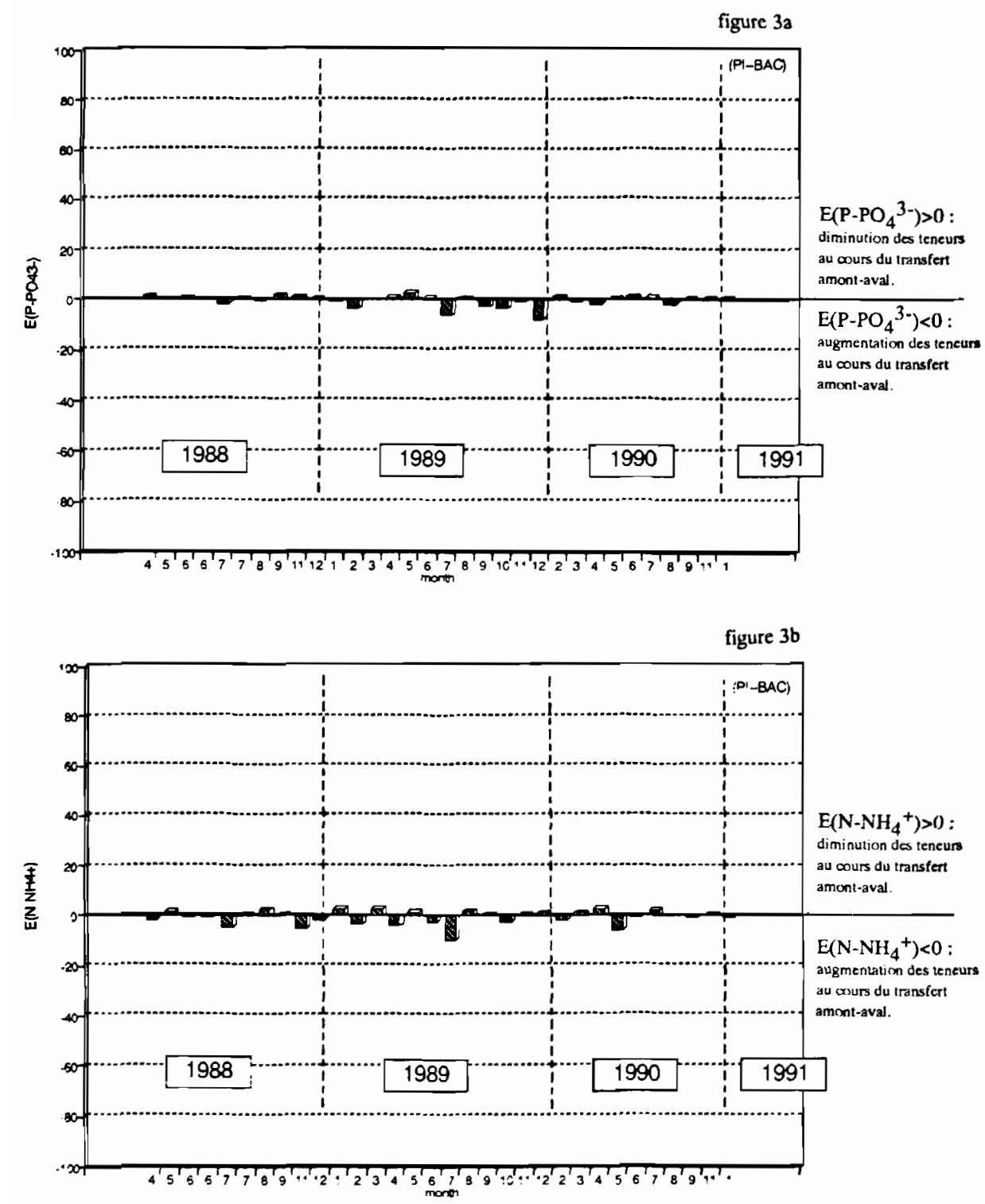

Fig. 3. - Capacité épuratrice $E\left(P O_{4}^{-}\right)$(a) et $E\left(N_{4}\right)$ (b) du Rhin canalisé.

Fig. 3. - Self purification capacity $\mathrm{E}\left(\mathrm{PO}_{4}^{3}\right)$ (a) and $\mathrm{E}\left(\mathrm{NH}_{4}\right)$ (b) of the canalised Rhine. 
optimise les conditions de relargage), ou à des paramètres hydrologiques. Nous avons en effet observé une importante hausse des teneurs en phosphates et $\mathrm{DBO}_{5}$ en décembre 1989, à la suite d'un épisode de très basses eaux (débit $<400 \mathrm{~m}^{3} \cdot \mathrm{s}^{-1}$ ). Un tel étiage provoque immanquablement un accroissement de la concentration des éléments en solution.

\subsection{Les Vieux-Rhin}

\subsubsection{Le Vieux-Rhin à hauteur de Rhinau}

\subsubsection{Evolution amont-aval et comportement épurateur}

Le Vieux-Rhin de Rhinau présente dans sa partie amont (fig. 4) une amorce de capacité épuratrice vis-àvis des phosphates notamment. Cette aptitude épuratrice peut s'exalter à certaines périodes (septembre 1990 notamment). Ceci est à mettre en relation avec la colonisation des eaux par une flore macrophytique encore eurytope certes, mais déjà plus structurée et plus diversifiée que celle présente dans le Rhin canalisé (Eglin et Robach, 1992). Cette épuration ne semble pas concerner la charge en azote ammoniacal, ce qui pourrait s'expliquer par le fait que les processus physiques de désorption des nutriments à partir de la phase solide, (sédiment et particules en suspension), sont optimisés par certains paramètres hydrologiques (que présente le Vieux-Rhin): faible profondeur, présence de sédiments qui s'accumulent en raison d'un faible débit mais qui sont facilement remis en suspension lors d'épisodes venteux ou orageux. Une étude bactériologique, au niveau des phases aqueuses et de la phase du sédiment, serait à prévoir pour déterminer la part d'une éventuelle réduction bactérienne des nitrates en ammoniaque, peu probable dans la phase aqueuse en raison des teneurs en oxygène généralement très élevées (taux de saturation excédant souvent $100 \%$ ).

Par ailleurs, le Vieux-Rhin offre des conditions attrayantes pour le développement du phytoplancton (eaux calmes, faible profondeur induisant un réchauffement rapide des eaux en période estivale, forte charge en éléments eutrophisants...). On peut de ce fait concevoir, que le Vieux-Rhin se comporte comme un "nid à plancton" à certaines périodes de l'année (il suffit pour s'en convaincre d'observer la couleur brun-rouge des eaux du Vieux-Rhin au printemps, couleur qui témoigne du développement "explosif " de diatomées. Cette hypothèse est étayée également par les valeurs de la $\mathrm{DBO}_{5}$ (tableau IV) qui montrent une augmentation quasi-régulière entre le point $V \times 1$ et le point $V \times 5$.

Or les populations de micro-organismes se développent et croissent rapidement, entrainant un recyclage rapide des éléments minéraux (cf $E\left(P_{4}\right)=70$, en juillet 1989 entre $V \times 1$ et $\mathrm{V} \times 2$ ). Mais, inversement, une période de dégénérescence peut se traduire temporairement par une libération massive de matière organique, entrainant dans un second temps une augmentation de la charge en azote ammoniacal. Cette hypothèse 
figure 4a

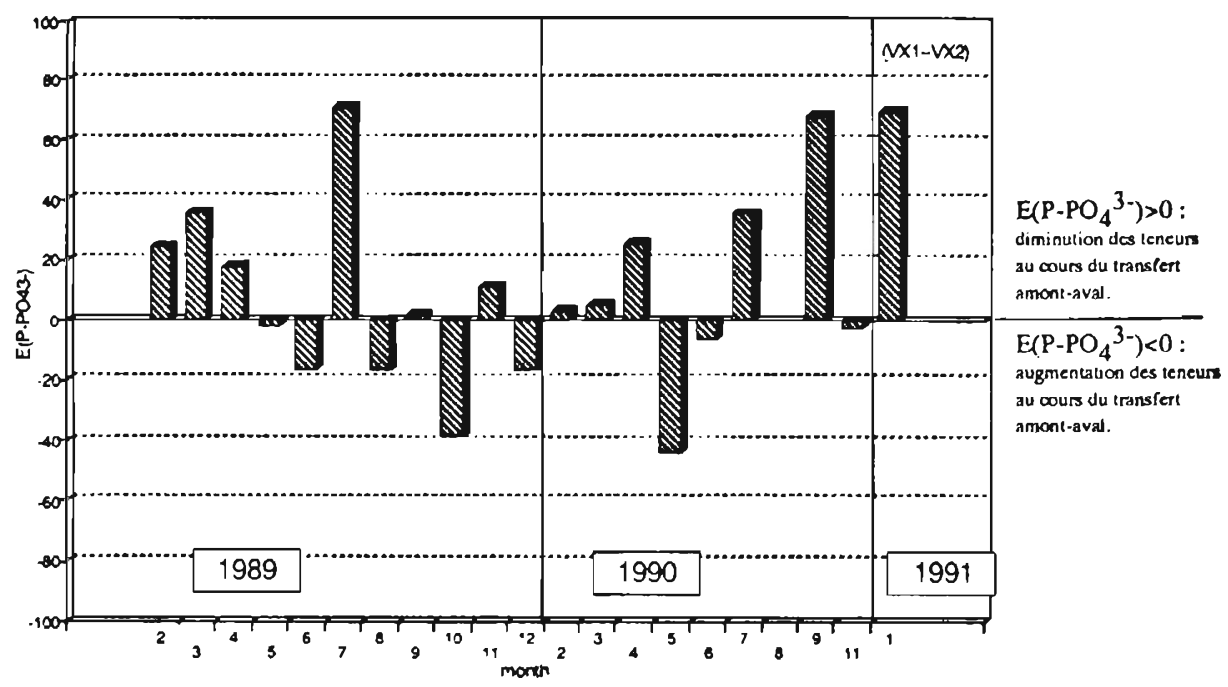

figure $4 b$

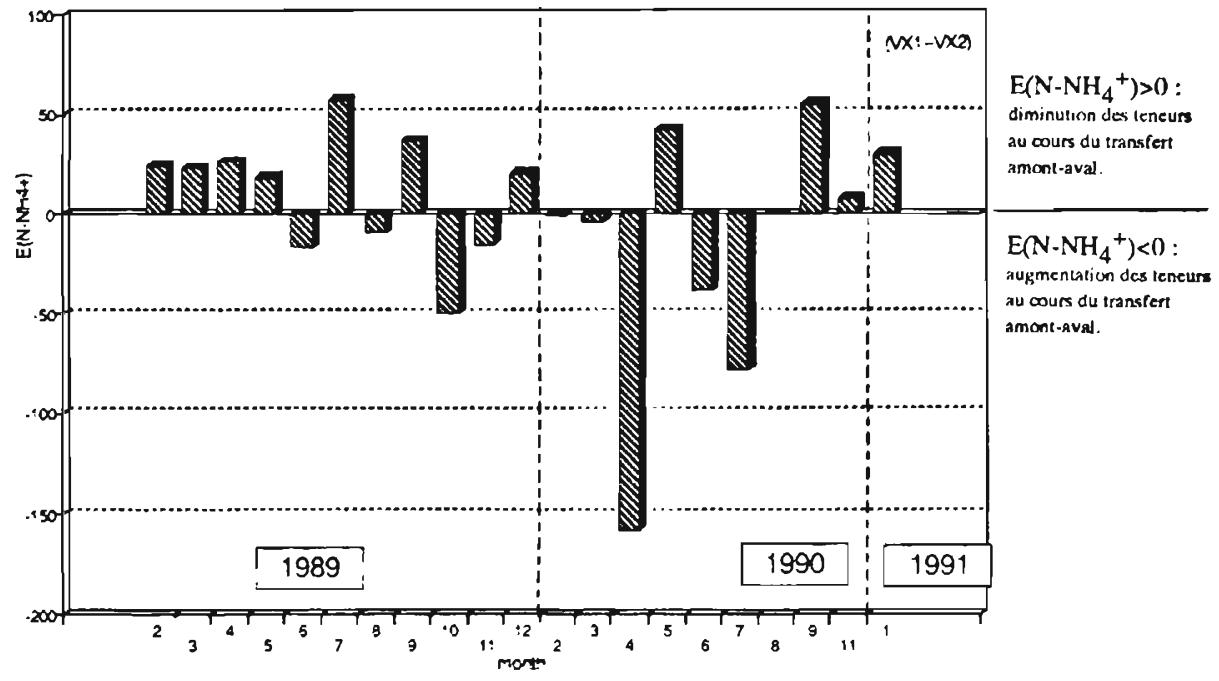

Fig. 4. - Capacité épuratrice $E\left(\mathrm{PO}_{4}^{3}\right)$ (a) et $E\left(\mathrm{NH}_{4}\right)$ (b) du Vieux-Rhin amont (Rhinau).

Fig. 4. - Self purification capacity $E\left(P O_{4}^{3}\right)$ (a) and $E\left(\mathrm{NH}_{4}\right)$ (b) of the upstream part of the "OldRhine" (Rhinau). 
Tableau IV. - Moyennes estivales de la $\mathrm{DBO}_{5}$ et évolution amont-aval dans le Vieux-Rhin. Table IV. - Vegetative average values of $\mathrm{BOD}_{5}$ and upstream-downstream evolution in the Old-Rhine.

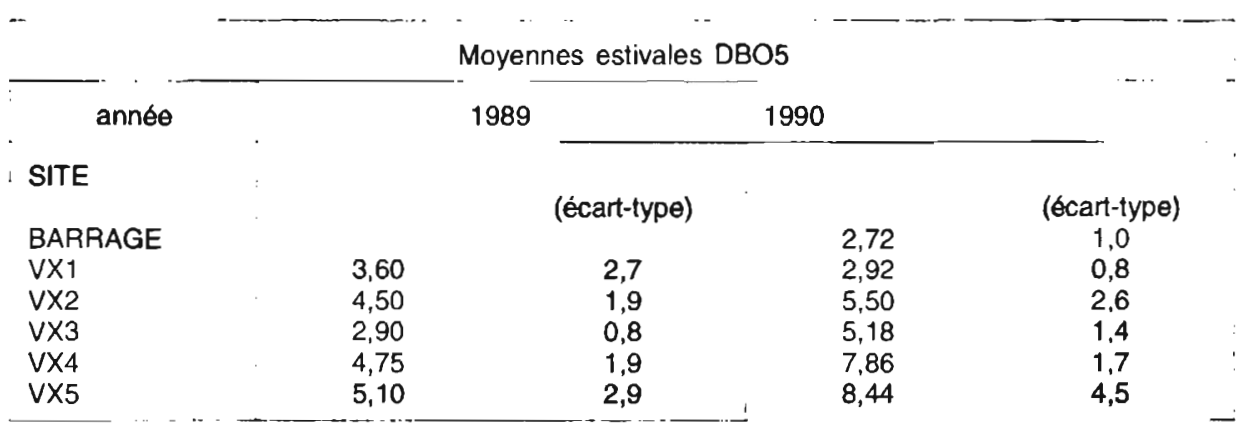

est à mettre en relation avec les fortes teneurs en oxygène dissous du VieuxRhin (production par photosynthèse pouvant induire des sursaturations), et avec les teneurs en Chlorophylle a qui $y$ dépassent quelquefois $50 \mu \mathrm{g} \cdot \mathrm{I}^{-1}$.

En ce qui concerne les phosphates, la capacité épuratrice $\left(E\left(P O_{4}\right)\right.$ ) entre les points $V \times 1$ et $V \times 2$ distants de $1,2 \mathrm{~km}$ environ, est globalement positive sur les trois années:

$E\left(\mathrm{PO}_{4}\right)=11 \quad \mathrm{du} \quad 24.02 .89 \quad \mathrm{au}$ 09.01 .91 (20 valeurs).

En ce qui concerne la charge ammoniacale, la capacité épuratrice $\left(\mathrm{E}\left(\mathrm{NH}_{4}\right)\right.$ est globalement faiblement négative $(-2)$ pour la même période:

Les comparaisons statistiques des moyennes amont-aval, n'accordent pas une importance significative (au seuil de $5 \%$ ) aux variations globales des teneurs en azote ammoniacal.

Si le Vieux-Rhin présente une activité épuratrice encore globalement médiocre, cette dernière peut néan- moins présenter des périodes particulièrement efficaces, comme cela fut le cas en juillet et septembre 1989, mois pendant lesquels la charge en phosphates diminue de plus de $50 \%$ en moins d'un kilomètre (entre $\mathrm{V} \times 1$ et $\mathrm{V} \times 2)$.

Mais cette capacité épuratrice potentielle est malheureusement compromise au niveau de l'île de Rhinau. En effet, à la hauteur du second seuil bétonné (à $3 \mathrm{~km}$ environ en aval du barrage), l'activité épuratrice naissante est toujours compromise par des apports hyper-eutrophes en provenance du Canal Leopold (fig. 5).

Ces perturbations sont responsables sur l'ensemble du parcours du Vieux-Rhin (11 km environ) d'un rendement épurateur globalement négatif sur les trois années d'études (20 campagnes de prélèvement):

$$
\begin{aligned}
& \left.E\left(\mathrm{PO}_{4}\right)=-0,29 \text { (entre } \mathrm{V} \times 1 \text { et } \mathrm{V} \times 5\right) \\
& E\left(\mathrm{NH}_{4}\right)=-0,37 \text { (entre } \mathrm{V} \times 1 \text { et } \mathrm{V} \times 5 \text { ). }
\end{aligned}
$$




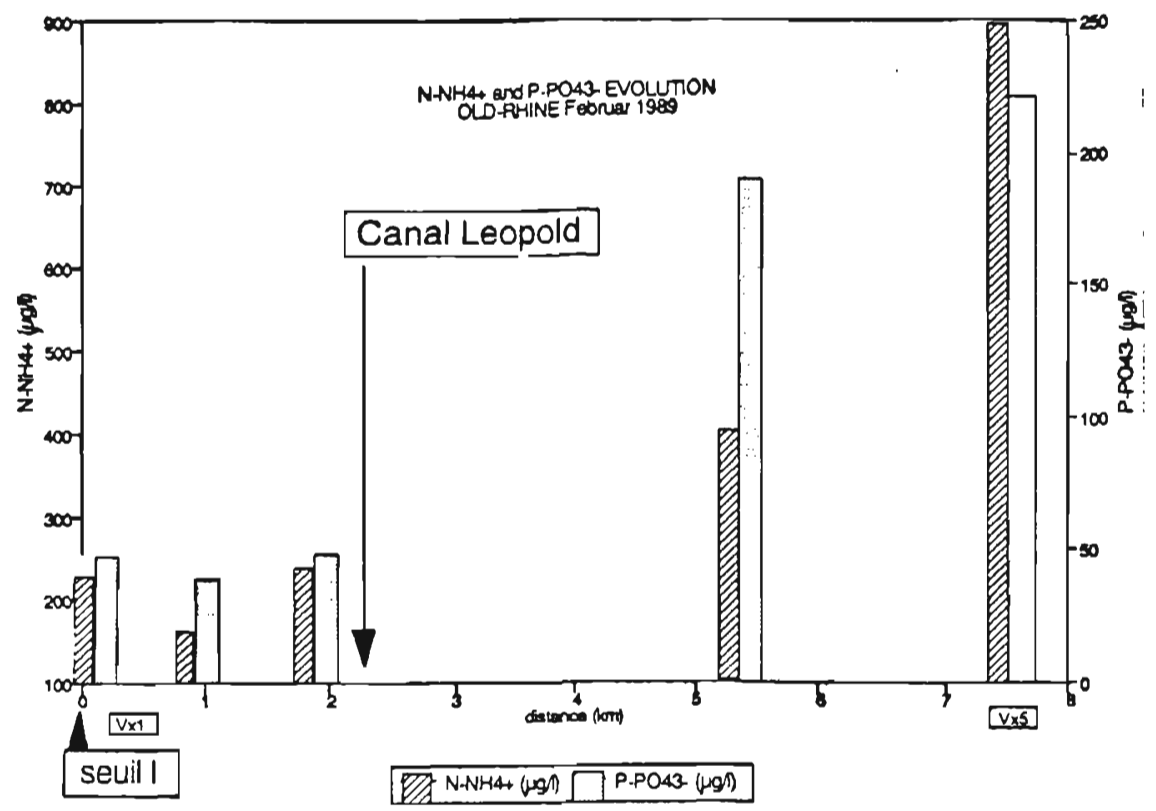

Fig. 5. - Evolution des teneurs en $\mathrm{PO}_{4}^{3-}$ ef $\mathrm{NH}_{4}^{+}$dans le Vieux--Rhin (Rhinau).

Fig. 5. - $\mathrm{PO}_{4}^{3}$ and $\mathrm{NH}_{4}$ evolution in the "Old-Rhine" (Rhinau).

Dans la partie aval du Vieux-Rhin, contaminée par le Canal Leopold, la hausse quasi systematique et souvent spectaculaire des valeurs de la $\mathrm{DBO}_{5}$ et de la Chlorophylle a, est imputable aux déversements considérables de phosphates (pics de plus de $2000 \mu \mathrm{g} \cdot \mathrm{l}^{-1}$ de $\mathrm{P}_{-} \mathrm{PO}_{4}^{3-}$ et $\left.\mathrm{N}-\mathrm{NH}_{4}^{+}\right)(\mathrm{Cf}$ : relation directe entre teneurs en phosphore dans l'eau et teneurs en Chlorophylle a, Barbe 1981).

Après le déversement des eaux contaminées, le Vieux-Rhin reprend ses fonctions épuratrices, qui sont globalement positives, mais néanmoins irrégulières en raison des contamination anthropiques (fig. 6). Malheureusement, ces capacités épu- ratrices naissantes ne pourront se développer en raison de la proximité de la confluence du Vieux-Rhin avec le Rhin canalisé. Le Vieux-Rhin, potentiellement épurateur mais dont les capacités épuratrices sont limitées par des perturbations anthropiques, délivrera donc dans le Rhin canalisé, des eaux plus eutrophes et plus polluées qu'il n'en avait héritées à l'amont de l'île.

\subsubsection{Evolution saisonnière}

En ce qui concerne les phosphates et l'azote ammoniacal, l'étude des variations saisonnières révèle une qualité d'eau supérieure (au point $V X 1$, donc avant la zone de perturbations) au cours des périodes végé- 

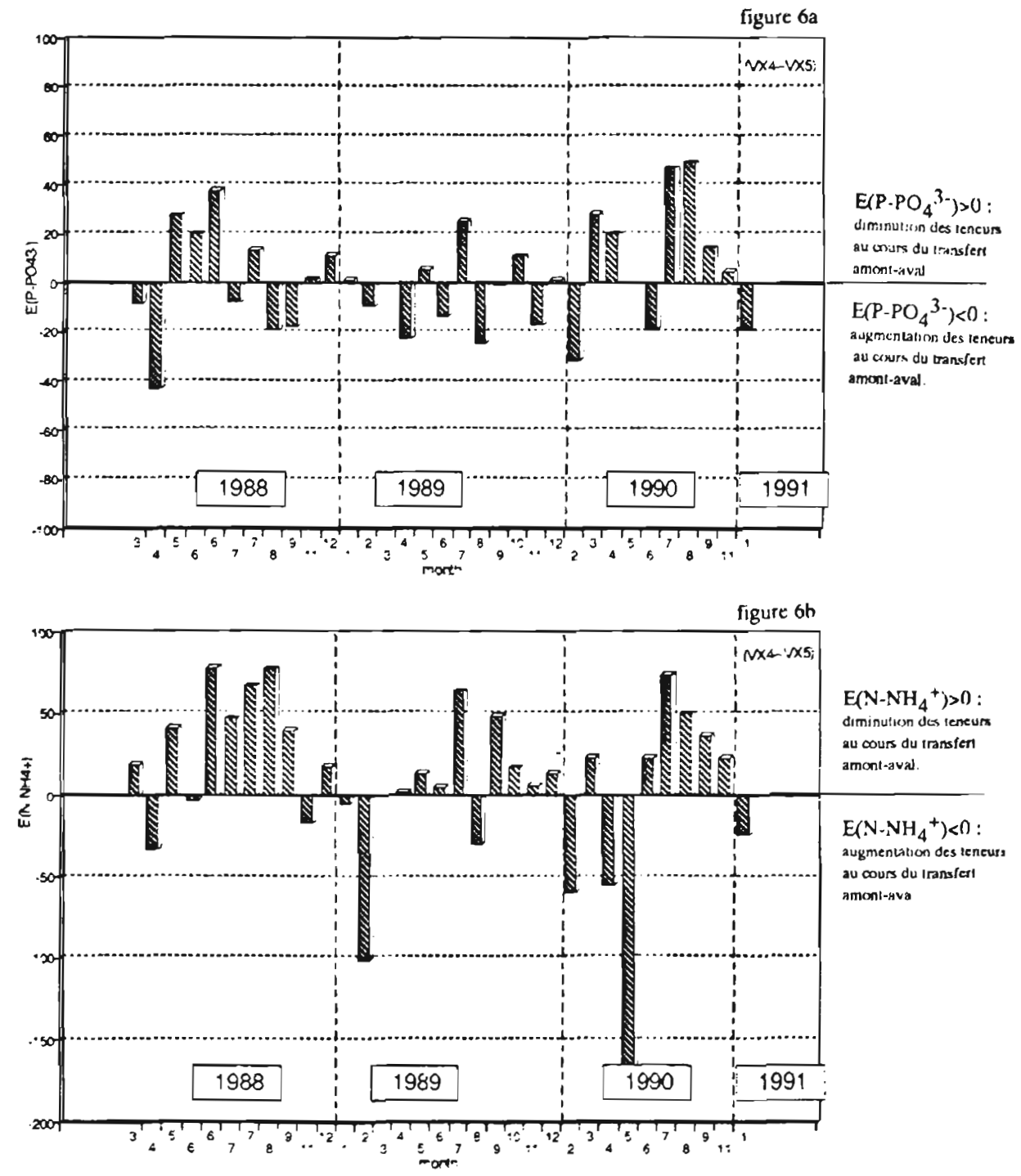

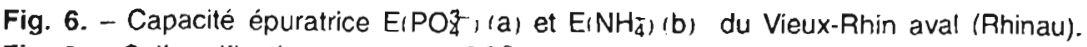

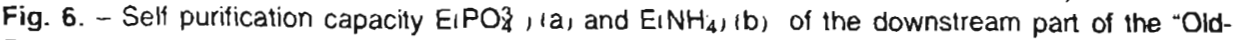
Rhine" (Rhinau). 
tatives, par rapport aux périodes non végétatives. En effet, au cours des périodes végétatives (année 1989 et 90 confondues), la moyenne des teneurs en phosphates était de $37 \mu \mathrm{g} \cdot \mathrm{l}^{-1} \quad$ (Ec. Type $=8$ ) contre 49 $(E c$. Type $=18)$ en période non végétative, tandis que les moyennes respectives en azote ammoniacal étaient de $107 \mu \mathrm{g} \cdot \mathrm{l}^{-1} \quad$ (Ec.type $\left.=57\right)$ contre 219 (Ec.type $=61$ ). Les différences sont significatives au seuil de $5 \%$.

Cette amélioration de la qualité de l'eau en période estivale, (alors même, comme nous l'avons déjà vu, que cette période coincide avec une optimalisation des phénomènes de désorption des nutriments à partir du sédiment), est donc à rattacher au moindre degré d'artificialisation de ce système aquatique, ce qui permet l'installation d'une vie macrophytique et planctonique plus structurée.

\subsubsection{Le Vieux-Rhin à Gerstheim}

Cette potentialité épuratrice, optimalisée en période végétative, et non troublée par des apports anthropiques, se retrouve de la même manière dans le Vieux-Rhin à hauteur de l'île de Gerstheim entre les points G1 et Gl (fig. 7).

La capacité épuratrice des charges phosphatées et ammoniacales semble particulièrement élevée en période végétative 1989 , période qui n'a pas été troublée par une crue eutrophisante:

$$
E\left(\mathrm{PO}_{4}^{3-}\right)=34 \text { et } E\left(\mathrm{NH}_{4}^{+}\right)=39
$$

Par contre, cette même capacité semble sévèrement compromise en période végétative, vis-à-vis des phosphates notamment, après la crue de février 1990. Le comportement épurateur global, pour 1989 et 1990, est donc sensiblement plus faible $E\left(P_{4}\right)=13$ et $E\left(N_{4}^{+}\right)=33$, en période végétative.

\subsection{Les bras tressés forestiers connectés au Vieux-Rhin}

\subsubsection{Le Schollengiessen de l'île de Gerstheim}

Rappellons que le Schollengiessen (débit $=1 \mathrm{~m}^{3} \cdot \mathrm{s}^{-1}$ environ) est caractérisé par sa faible profondeur, par son lit quasi-exclusivement graveleux, par la fréquence de seuils à courant vif et par une faible biomasse végétale aquatique.

Notons également que ce bras tressé est perturbé:

- d'une part, par des apports phréatiques infra-aquatiques diffus (part non encore estimée) à la hauteur du point G3a. Ces apports d'eaux catharobes et méso-eutrophes contribuent, par dilution, aux processus globaux d'amélioration de la qualité de l'eau.

- d'autre part, par deux prises d'eaux directes sur le Vieux-Rhin (débit $\left.<0,5 \mathrm{~m}^{3} \cdot \mathrm{s}^{-1}\right), 300 \mathrm{~m}$ environ après le point G3a.

De ce fait, nous ne considèrerons dans cette étude, que l'évolution des paramètres entre les points $\mathrm{G} 1$ et $\mathrm{G} 3$, distants de $800 \mathrm{~m}$ environ. Ce bras tressé forestier, faiblement végétalisé se caractérise par un comportement 
figure 7a

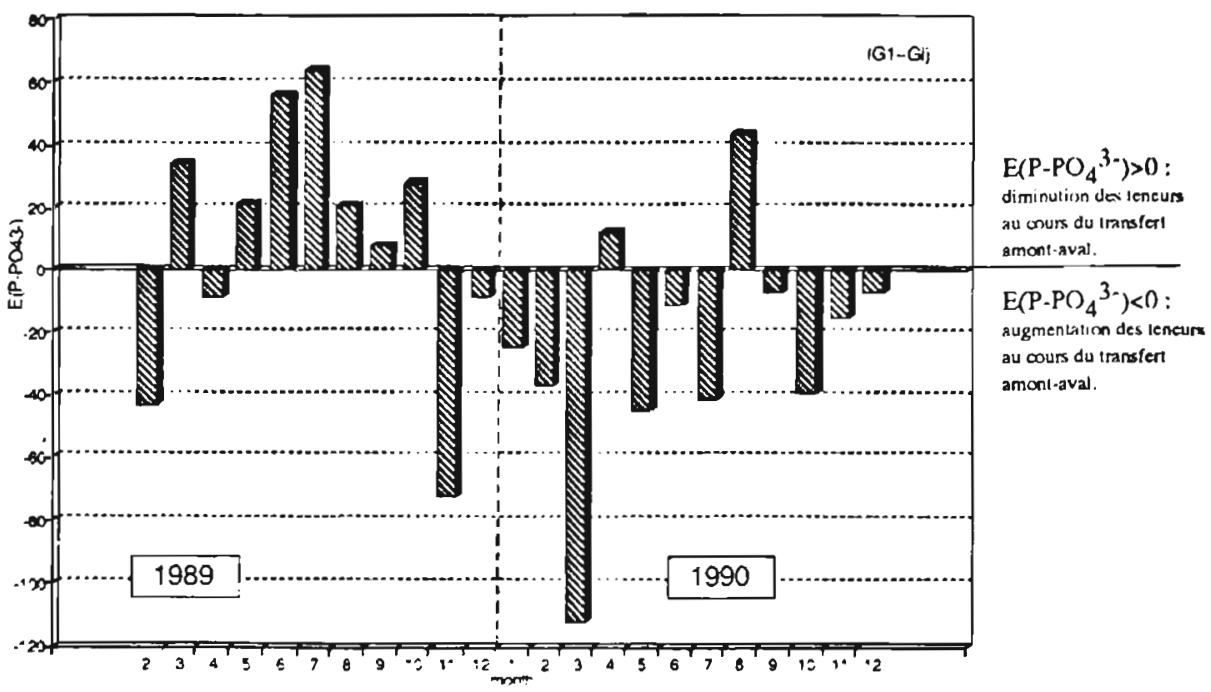

figure $7 \mathrm{~b}$

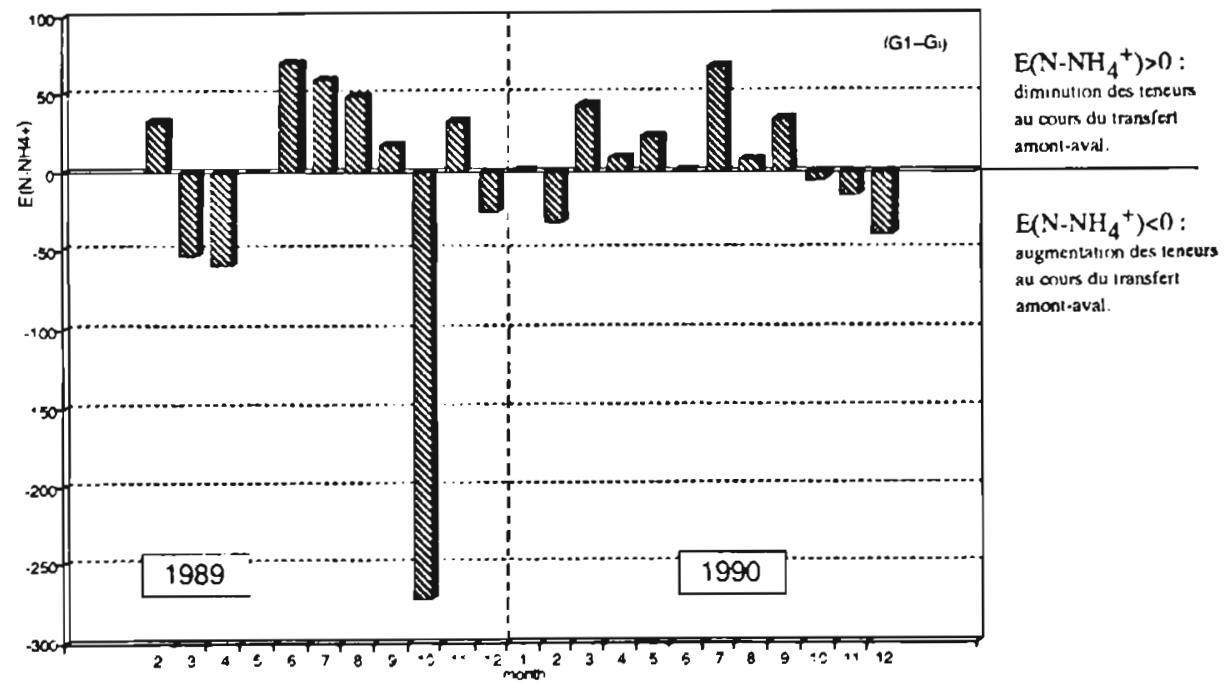

Fig. 7. - Capacité épuratrice $E\left(\mathrm{PO}_{4}^{3}\right)$ (a) et $E\left(\mathrm{NH}_{4}\right)$, b) du Vieux-Rhin (Gerstheim).

Fig. 7. - Self purification capacity $E\left(\mathrm{PO}_{4}^{-2}\right.$ ) (a) and $E\left(\mathrm{NH}_{4}\right)$ (b) in the "Old-Rhine" (Gerstheim). 


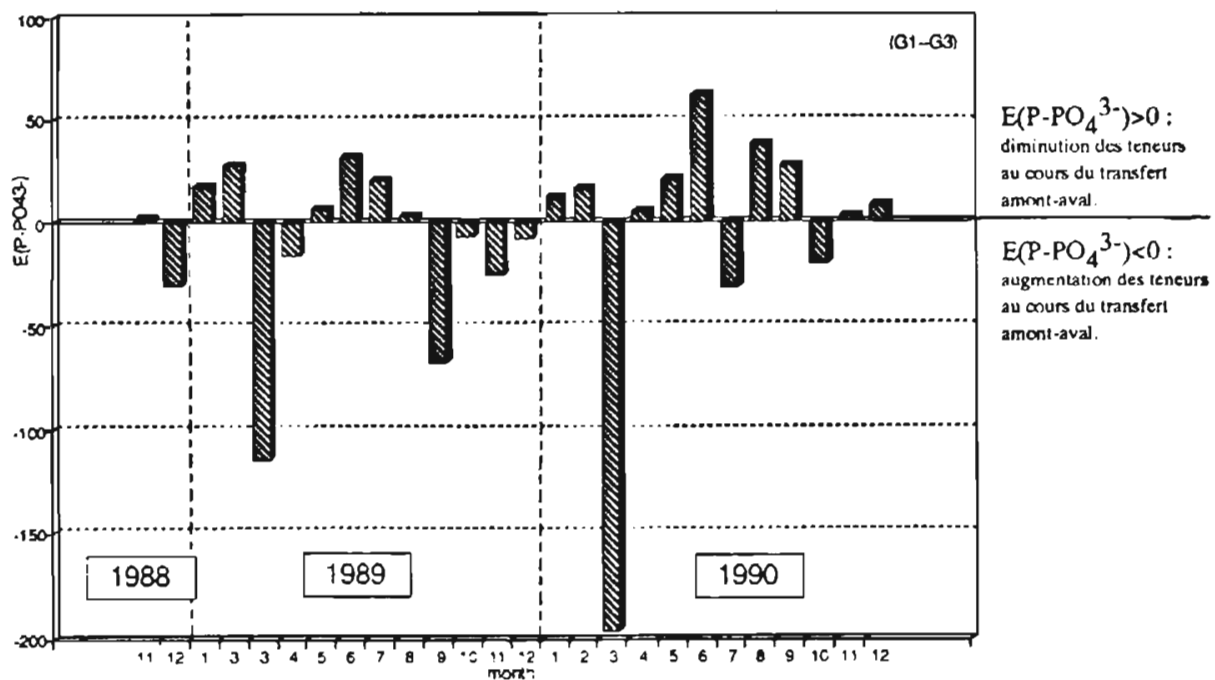

Pi,ure 8b

$\mathrm{E}\left(\mathrm{N}-\mathrm{NH}_{4}{ }^{+}\right)>0$ :

diminution des tencurs

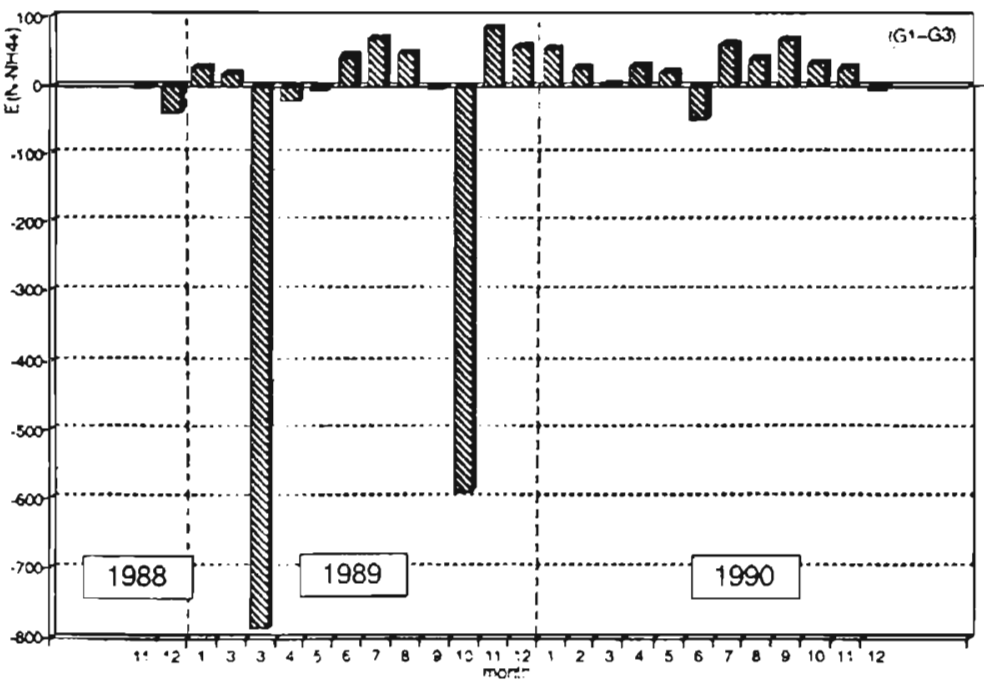

au conur du Iransfert amont-aval.

$\mathrm{E}\left(\mathrm{N} \cdot \mathrm{NH}_{4}{ }^{+}\right)<0$ : augmentation des teneurs au cours du transfert amont-ava

Fig. 8. - Capacité épuratrice $E\left(\mathrm{PO}_{4}^{3-}\right)$ (a) et $E\left(\mathrm{NH}_{4}\right)$ (b) du Schollengiessen (Gersheim). Fig. 8. - Self purification capacity $E\left(\mathrm{PO}_{4}^{3}\right)$ (a) and $E\left(\mathrm{NH}_{4}\right)$ (b) in the Schollgengiessen (Gerstheim). 
épurateur irrégulier (fig. 8), globalement, mais faiblement, positif en période végétative (1989 et 1990):

$$
E\left(P_{4}\right)=11,6 \quad E\left(N_{4}\right)=28,2
$$

Par contre, les capacités épuratrices sont négatives en période non végétative:

$$
E\left(P_{4}\right)=-19,8 \quad E\left(N_{4}\right)=-68,6 .
$$

Les périodes particulièrement défavorables en ce qui concerne l'évolution des teneurs en azote ammoniacal, sont généralement des périodes "pré " ou "post" végétatives (mars-avril-mai 1989 et octobre 1989 notamment). Ces périodes sont caractérisées respectivement, par la reprise de la décomposition de la matière organique végétale déposée en automne, mais différée par le froid hivernal, et d'autre part par les premières étapes automnales de cette décomposition.

\subsubsection{Le Schaftheu de l'île de Rhi- nau}

Ce cours d'eau est alimenté (comme le Schollengiessen à Gerstheim) par une prise d'eau directe sur le VieuxRhin, qui comme nous l'avons remarqué plus haut, est susceptible d'être temporairement affectée par les remous hypertrophes du canal Leopold. De ce fait, il subit plusieurs fois par an, une sévère aggravation de la qualité de ses eaux, ce qui, naturellement, tend à limiter ses capacités épuratrices globales.

II est possible d'individualiser deux portions dans ce cours d'eau:
- la portion amont, (moitié de la longueur totale) fortement végétalisée, de profondeur moyenne généralement inférieure à $2 \mathrm{~m}$, présente un fond essentiellement limono-vaseux.

- la portion aval, parcourt la seconde moitié de l'île de Rhinau sur trois $\mathrm{km}$ environ. Celle-ci recevait, par contact direct, jusqu'à la fin de l'année 1988 des apports importants d'eau en provenance du Vieux-Rhin, à la hauteur du point $\mathrm{SCH} 6$ (cette communication a été fortement limitée depuis, par la mise en place d'un rideau de palplanches). Cette fraction est non végétalisée, pour des raisons non encore clairement explicitées, et présente un fond également essentiellement limono-vaseux. Cette partie débouche, à hauteur du point $\mathrm{SCH} 8$ dans le Vieux-Rhin.

Ces deux portions du Schaftheu présentent des comportements épurateurs très différents que nous exposerons successivement.

\subsubsection{Tronçon non végétalisé}

L'évolution amont-aval des charges eutrophisantes et polluantes a été plus particulièrement suivie dans une portion morphologiquement homogène, définie par les points $\mathrm{SCH} 7$ et SCH8 (fig. 9), distants de $2,8 \mathrm{~km}$ environ. Cette étude a débuté en août 1989.

Le comportement épurateur de ce tronçon est globalement positif sur les trois années d'étude avec des périodes particulièrement favorables ainsi qu'en témoigne une réduction considérable de $1680 \mu \mathrm{gP} . \mathrm{l}^{1}$ en février 1990 entre ces deux points. 


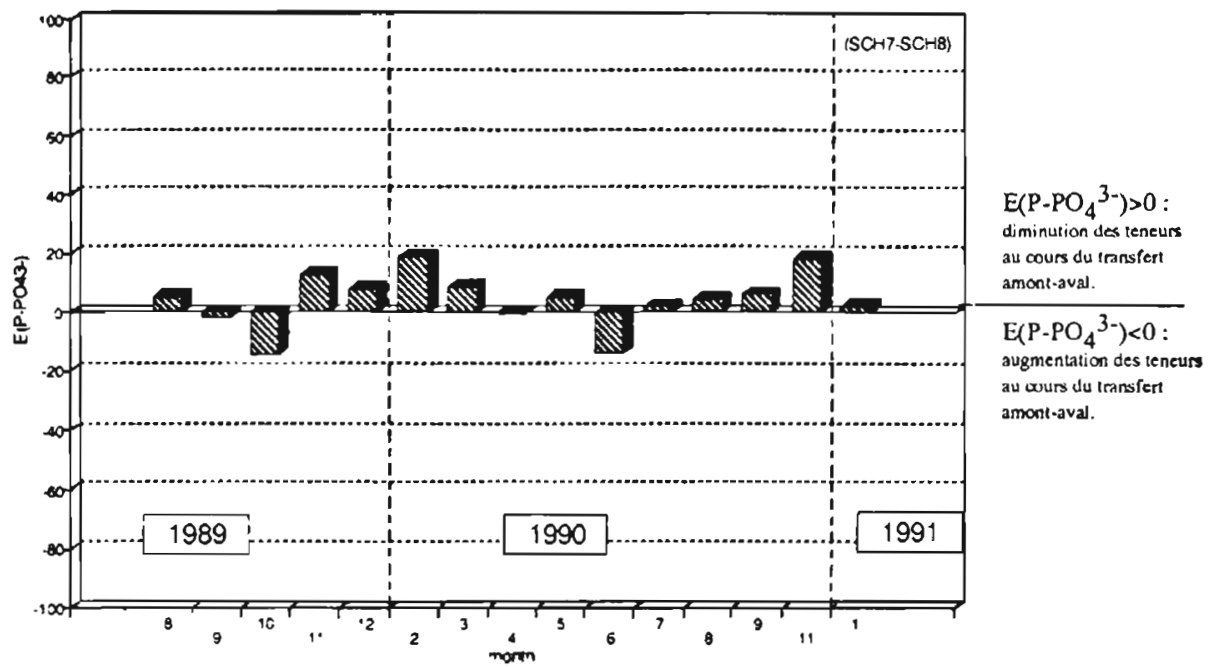

Fiar.t. in

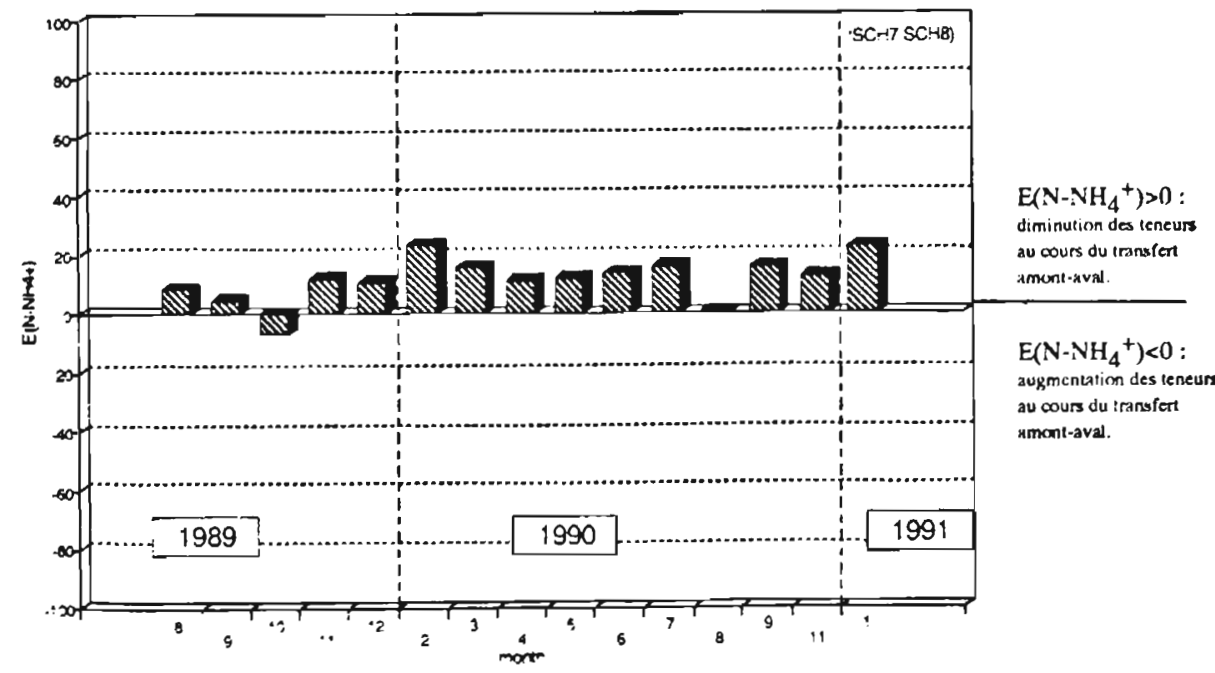

Fig. 9. - Capacité épuratrice $E\left(\mathrm{PO}_{4}^{3}\right)$ (a) et $E\left(\mathrm{NH}_{4}\right)$ (b) du Schaftheu aval (non végétalisé). Fif. 9. - Self purification capacity $\mathrm{E}\left(\mathrm{PO}_{4}^{3}\right)$ (a) and $\mathrm{E}\left(\mathrm{NH}_{4}\right)$ (b) in the downstream part of the Schaftheu (unvegetalised area). 
Mais la capacité épuratrice diminue paradoxalement en période estivale: $\mathrm{E}\left(\mathrm{PO}_{4}\right)=1,0$ en période estivale contre 6,0 en période non végétative, $\mathrm{E}\left(\mathrm{NH}_{4}\right)=10,2$ en période estivale contre 11,4 en période non végétative.

Notons également, que la canopée forestière forme une voûte dense audessus du cours d'eau, ce qui limite l'activité photosynthétique, tant algale que macrophytique. Ceci est susceptible d'expliquer en partie le fait que cette fraction du Schaftheu présente toujours des eaux relativement claires, jamais colorées par les diatomées comme celles peu végétalisées du Vieux-Rhin.

D'autre part, les comptages bactériens ne signalent pas d'anomalie particulière, par rapport à la fraction amont du Schaftheu $\left(2,5 \cdot 10^{2}\right.$ à $1,2 \cdot 10^{4}$ bactéries par $\mathrm{ml}$ ). II faut noter à ce sujet, que les teneurs en nitrates de la fraction aval du Schaftheu, entre le point $\mathrm{SCH} 7$ et le point $\mathrm{SCH} 8$ présentent de façon quasi systématique une augmentation (même légère), ce qui, lié à la diminution des teneurs en azote ammoniacal, pourrait traduire une activité bactérienne nitrifiante (oxydation bactérienne de l'ammoniaque en nitrate). Cette hypothèse reste cependant à confirmer.

Nous attribuons par conséquent l'efficacité amoindrie des capacités épuratrices en période estivale,

- d'une part, aux relargages plus importants de nutriments en période estivale qu'en période non estivale.

- d'autre part, à l'absence de macrophytes aquatiques (qui par absorp- tion auraient contribué à réduire les charges en éléments eutrophisants), et à la faible biomasse planctonique (teneur en chlorophylle $\mathrm{a}<18 \mu \mathrm{g} \cdot \mathrm{I}^{-1}$ ),

Par ailleurs les résultats montrent que l'activité épuratrice de la portion aval du bras forestier du Schaftheu est moins efficace vis-à-vis des phosphates qu'en ce qui concerne la charge ammoniacale, même si la réduction de la charge phosphatée peut, par moment, atteindre des valeurs élevées (réduction de $160 \mu \mathrm{g} \cdot \mathrm{I}^{-1}$ en février 1990). Hormis ces rares périodes particulières, les moyennes des teneurs en phosphates des points $\mathrm{SCH} 7$ et $\mathrm{SCH}$, n'apparaissent pas significativement différentes (au seuil de $5 \%$ ).

Les mesures de phosphore total dans le sédiment, ne révèlent pas d'enrichissement particulier dans cette zone par rapport au Schaftheu amont.

Si la capacité épuratrice globale nous apparaît essentiellement liée aux phénomènes physiques concernant les transferts de nutriments dans les différentes phases d'un écosystème aquatique d'une part, et aux activités bactériennes d'autre part, la part relative de ces deux processus reste, elle aussi à déterminer.

\subsubsection{Tronçon végétalisé}

Au cours du transfert amont-aval, des modifications dans la composition végétale corrélées à des modifications sensibles de la qualité de l'eau, nous ont conduites à distinguer au sein de ce tronçon, deux secteurs présentant des comportements épurateurs distincts : 
- les 800 premiers mètres où l'épuration est effective,

- le secteur situé en aval, où l'épuration est compromise en raison de contaminations ponctuelles en phosphate et ammoniaque. En effet, la qualité de l'eau au niveau du site $\mathrm{SCH} 5 \mathrm{a}$ se détériore de façon significative puisque des pics de phosphates très localisés sont régulièrement enregistrés. A ce niveau, le Rhin canalisé est très proche du Schaftheu (moins de $20 \mathrm{~m}$ ). De plus, le tracé du fleuve canalisé coupe un ancien bras de tresse, qui alimentait le schaftheu avant les travaux de canalisation. Toutes ces observations permettent de supposer une contamination du Schaftheu par le Rhin canalisé, par l'intermédiaire de fuites souterraines captées par l'ancien chenal de tresse.

- évolution amont-aval entre les points $\mathrm{SCH} 2$ et $\mathrm{SCH} 5(0,8 \mathrm{~km})$

En moyenne, sur les trois années d'étude, nous observons une diminution de $12,7 \%$ des teneurs en phosphates entre le point $\mathrm{SCH} 2$ et le point $\mathrm{SCH} 5$ (les moyennes amont-aval sont significativement différentes au seuil de $5 \%, t=2,292)$. Cette épuration est maximalisée en période végétative $(21 \%$ de réduction en moyenne contre $3,7 \%$ en période non végétative).

La capacité épuratrice $E\left(\mathrm{PO}_{4}\right)$ a été particulièrement remarquable en été 1989 , où elle présente une moyenne de 35,2 (avec des pointes ponctuelles de 70 en août 1989). Notons d'ailleurs que cette capacité atteignit la valeur maximale de 84 en septembre 1988 , ce qui correspondait à une réduction de $300 \mu \mathrm{g} \cdot \mathrm{I}^{-1}$ en moins d'un $\mathrm{km}$. Cette capacité épuratrice globalement positive sur l'ensemble des trois années, est supérieure à celle relevée dans la partie non végétalisée du Schaftheu.

Vis-à-vis de la charge en azote ammoniacal (fig. 10), la capacité épuratrice du Schaftheu est également positive $\left(E\left(\mathrm{NH}_{4}\right)=33,7\right.$ durant les périodes végétatives). Contrairement au tronçon aval qui présentait une augmentation des teneurs en azote nitrique, cette fraction végétalisée se caractérise par une notable diminution des teneurs en nitrates.

L'activité épuratrice positive concerne également les charges polluantes, comme cela est suggéré par l'évolution des teneurs de la DBO5. Cette épuration est optimisée en période estivale: $E(D B O 5)=26,9$ en période végétative.

Ce dernier résultat, corrélé à l'accroissement des capacités de rétention de la charge eutrophisante durant la période végétative, suggère ici la part prépondérante de la végétation aquatique (ensemble macrophytesépiphytes-micro-organismes planctoniques) dans les processus épurateurs de cette fraction du Schaftheu. La part active des populations planctoniques est confirmée par les mesures des teneurs en chlorophylles a, qui subissent de fortes fluctuations temporelles (de quelques $\mu \mathrm{gl}^{-1}$ à $130 \mathrm{gl}^{-1}$ ). Inversement, les comptages bactériens, ne révèlent pas de fluctuations spatiales significatives; il ne semble pas que les populations 

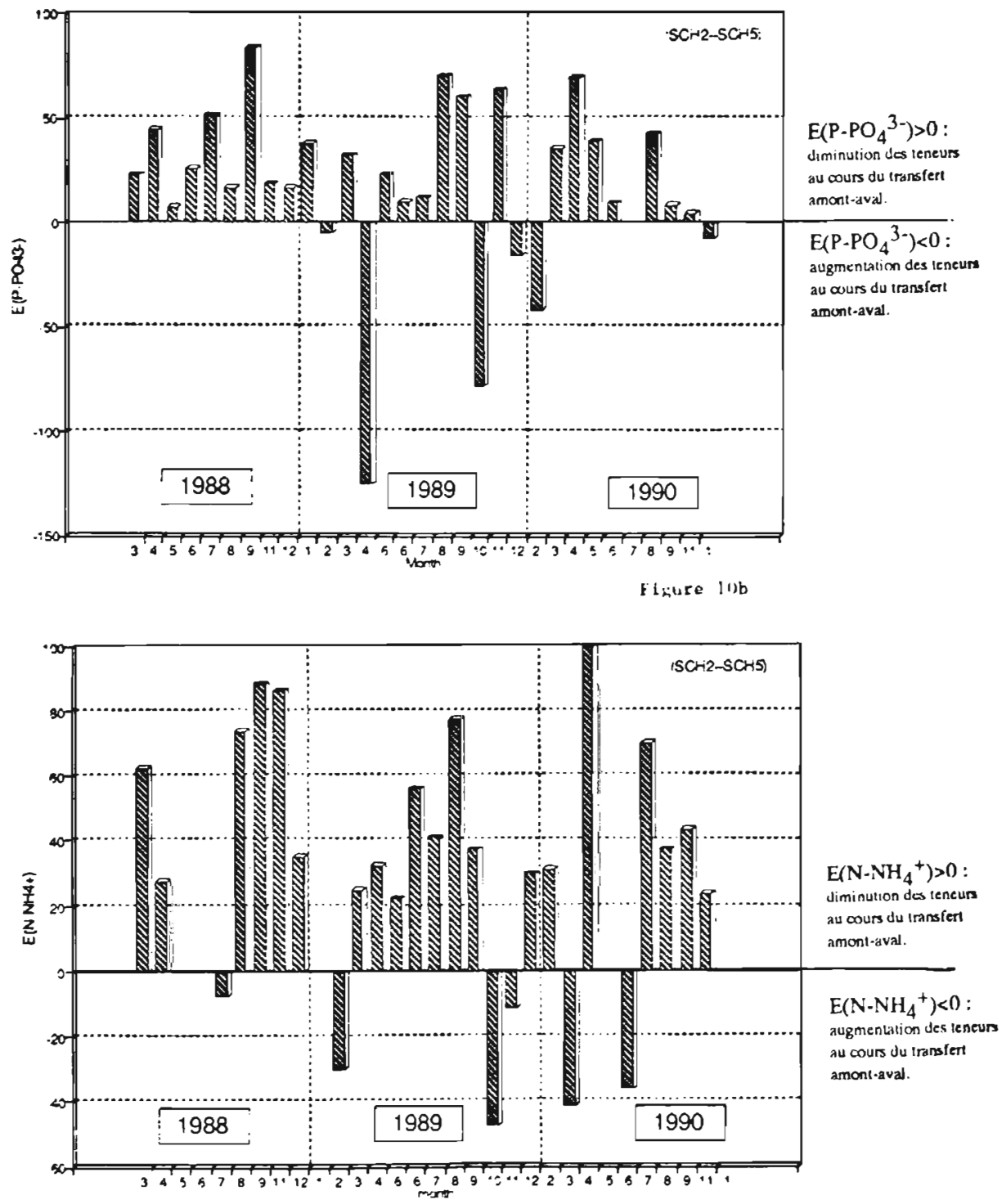

Fig. 10. - Capacité épuratrice $\mathrm{E}\left(\mathrm{PO}_{4}\right.$, (a) et $\mathrm{E}\left(\mathrm{NH}_{4}\right)$ (b) du Schaftheu amont (végétalisé). Fig. 10. - Self purification capacity $\mathrm{E}\left(\mathrm{PO}_{4}^{-}\right)$(a) and $\mathrm{E}\left(\mathrm{NH}_{4}\right)$ (b) in the upstream part of the Schaftheu (vegetalised area). 
bactériennes jouent un rôle prépondérant dans cette épuration.

Notons enfin, que la partie amont du Schaftheu, reçoit des eaux phréatiques, localisées et ponctuelles d'une part, à hauteur du point $\mathrm{SCH} 4$, diffuses et infra-aquatiques d'autre part entre le point $\mathrm{SCH} 4$ et le point $\mathrm{SCH} 5$. Ces eaux phréatiques oligosaprobes et méso-eutrophes contribuent par dilution, aux processus globaux d'amélioration de la qualité des eaux de surface. Ces apports s'expriment in situ, par une légère variation de la température (diminution en été, et augmentation en hiver, en raison de la relative sténothermie de la nappe phréatique sous-jacente (11 à $13^{\circ}$ en moyenne), ainsi que par une diminution du taux d'oxygène dissous, en raison du déficit relatif en oxygène des eaux souterraines. Des jaugeages au moulinet, effectués à trois reprises au courant de l'année 1990, ont permis d'estimer que ces apports ne représentaient guère plus de $20 \%$ du débit total.

Au cours de ces trois années d'étude, le bras tressé forestier, dans le premier tiers amont de son cours, montre donc une capacité d'autoépuration et de rétention d'eutrophisants positive, qui est exaltée en période végétative où elle devient remarquable.

\section{DISCUSSION}

Le tableau $V$ (a.b.c) résume les principaux résultats obtenus au niveau des systèmes lotiques en ce qui concerne la réduction de la charge en $\mathrm{P}_{-} \mathrm{PO}_{4}^{3-}$ (estimation de la réduction de la charge eutrophisante) et la réduction de la charge en $\mathrm{N}-\mathrm{NH}_{4}^{+}$(qui nous permet également d'estimer l'efficacité des processus de réduction de la matière organique). Les valeurs $\mathrm{E}\left(\mathrm{P}-\mathrm{PO}_{4}^{3-}\right)$ et $\mathrm{E}\left(\mathrm{N}-\mathrm{NH}_{4}^{+}\right)$données dans ce tableau représentent des moyennes obtenues sur trois années consécutives de prélèvements (1988 à 1990), sauf pour le Schaftheu. En effet, le débit de ce dernier, a été doublé en fin d'année 1988, à la suite d'un réaménagement de la prise d'eau sur le Rhin. Afin de respecter une homogénéité au niveau des paramètres hydrologiques seuls les résultats obtenus en 1989 et 1990 ont été pris en compte dans ce tableau.

1) L'efficacité des processus d'amélioration de la qualité de l'eau est optimisée en période estivale dans les systèmes végétalisés, ce qui confirme le rôle prédominant des biocénoses aquatiques. Cette épuration est particulierement efficace alors même que des phénomènes antagonistes de relargage du phosphore sont favorisés en été. Le Schaftheu amont qui recèle une végétation plus abondante que sa partie aval, exprime en période estivale, une efficacité sensiblement supérieure en ce qui concerne la réduction de la charge en phosphore. Malheureusement, le point de référence "aval" $(\mathrm{SCH} 5)$ situé dans la partie végétalisée du Schaftheu, est fréquemment perturbé par des apports anthropiques en raison de "la forte densité" de pê- 
Tableau V. - Capacités épuratrices moyennes a) $E\left(\mathrm{PO}_{4}\right)$, b) $E\left(\mathrm{NH}_{4}\right)$ et c) $E\left(\mathrm{DBO}_{5}\right)$ des principales unités lotiques de Rhinau et Gerstheim.

Table V. - Average self purification capacities a) $E\left(P_{4}\right)$, b) $E\left(N_{4}\right)$ and c) $E\left(D_{B O}\right)$ of the main running waters systems (Rhinau and Gerstheim).

\section{Tableau Va}

Un.
Fonctionnelles
Rhin canalisé
Vieux-Rhin
Vieux-Rhin
Vieux-Rhin
Schollengiessen
Schaftheu
Schaftheu

$\begin{array}{cc}\text { ILE } & \text { SITES } \\ & \\ \text { RHINAU } & \text { PI-BAC } \\ \text { RHINAU } & \text { VX1-VX5 } \\ \text { GERSTHEIM } & \text { G1-GI } \\ \text { GERSTHEIM } & \text { G1-G3 } \\ \text { RHINAU } & \text { SCH7-SCH8 } \\ \text { RHINAU } & \text { SCH2-SCH5 }\end{array}$

DISTANCE (KM)

$\mathrm{E}\left(\mathrm{PO}_{4}\right) \mathrm{E}\left(\mathrm{PO}_{4}\right) n v$ Val. Extrêmes:

\begin{tabular}{rrrr}
23,5 & $-0,1$ & $-0,7$ & $-7,8$ to 3,16 \\
1,2 & 8,5 & $-3,0$ & -42 to 49 \\
1,2 & 10,0 & 12,2 & $-44,1$ to 70,8 \\
1,2 & 12,9 & $-22,7$ & $-112,6$ to 63,9 \\
0,8 & 11,6 & $-19,8$ & $-196,7$ to 61,6 \\
2,8 & 1,0 & 6,0 & $-14,6$ to 19 \\
0,8 & 31,1 & 4,7 & -125 to 84,2 \\
\hline
\end{tabular}

\section{Tableau Vb}

\begin{tabular}{lcc} 
Un. & \multicolumn{1}{c}{ ILE } & SITES \\
Fonctionnelles & & \\
$\begin{array}{l}\text { Rhin canalisé } \\
\text { Vieux-Rhin }\end{array}$ & & PHINAC \\
Vieux-Rhin & RHINAU & VX4-VX5 \\
Vieux-Rhin & RERSTHEIM & VX1-VX2 \\
Schollengies- & GE 1 -GI \\
sen & GERSTHEIM & G1-G3 \\
Schaftheu & RHINAU & SCH7-SCH8 \\
Schaftheu & RHINAU & SCH2-SCH5
\end{tabular}

DISTANCE (KM)

23,5
1,2
1,2
1,2
0,8
2,8
0,8

$\mathrm{E}\left(\mathrm{NH}_{4}\right) v \quad \mathrm{E}\left(\mathrm{NH}_{4}\right) \mathrm{nv}$

0,6

12,7

6,6

33,0

28,2

10,2

33,7

$\begin{array}{rc}0,0 & -9,8 \text { to } 3,15 \\ -13,6 & -168 \text { to } 79 \\ -8,7 & -157,5 \text { to } 56,9 \\ -29,3 & -272,7 \text { to } 70,5 \\ -68,6 & -788 \text { to } 84 \\ 11,4 & -7 \text { to } 23,5 \\ 20,2 & -47,5 \text { to } 100\end{array}$

\section{Tableau Vc}

\begin{tabular}{|c|c|c|c|c|c|c|}
\hline $\begin{array}{l}\text { In. } \\
\text { onctionnelles }\end{array}$ & ILE & SITES & $\begin{array}{l}\text { DISTANCE } \\
\text { (KM) }\end{array}$ & $E\left(D^{2} O_{5}\right) v$ & $E\left(D^{2} O_{5}\right) n v$ & Val. Extrêmes \\
\hline $\begin{array}{l}\text { leux-Rnin } \\
\text { ieux-Rhin } \\
\text { ieux-Rhin } \\
\text { chollengies- } \\
\text { en } \\
\text { chaftheu } \\
\text { chaftheu }\end{array}$ & $\begin{array}{c}\text { RHINAU } \\
\text { RHINAU } \\
\text { GERSTHEIM } \\
\text { GERSTHEIM } \\
\text { RHINAU } \\
\text { RHINAU }\end{array}$ & $\begin{array}{c}\text { XX1-VX2 } \\
\text { G1-G1 } \\
\text { G1-G3 } \\
\text { SCH7-SCH8 } \\
\text { SCH2-SCH5 }\end{array}$ & $\begin{array}{r}23,5 \\
1,2 \\
1,2 \\
1,2 \\
0,8 \\
2,8 \\
0,8\end{array}$ & $\begin{array}{r}-0,6 \\
-4,4 \\
-74,0 \\
-63,0 \\
-74,0 \\
-6.4 \\
26,9\end{array}$ & $\begin{array}{r}0,5 \\
-40,8 \\
-155,1 \\
-3,8 \\
-12,0 \\
9,6 \\
-1,4\end{array}$ & $\begin{array}{c}-14,6 \text { to } 4,26 \\
-83,3 \text { to } 46,2 \\
-697 \text { to } 24 \\
-370,7 \text { to } 67 \\
-655,2 \text { to } 125 \\
-38 \text { to } 26,8 \\
-212,8 \text { to } 125\end{array}$ \\
\hline
\end{tabular}

$v=$ période végétative

cheurs qui se traduit inévitablement par une pollution liée à toutes les activités halieutiques. II est donc probable que le rendement de l'autoépuration soit sous-estimé par rapport à la fraction non végétalisée du Schaftheu, qui, par un concours de circonstances, est d'un accès plus difficile (berges impraticables, forêt riveraine quasi impénétrable...). 
2) Ce sont les bras forestiers fortement végétalisés qui attestent d'une épuration remarquablement active même en période non estivale. Inversement, les systèmes aquatiques les plus artificialisés (et donc les moins végétalisés dans notre secteur d'étude) se comportent comme des systèmes quasi inertes vis-à-vis de l'épuration des eaux.

3) Si la réduction des teneurs en $P$ et $N$ est particulièrement efficace en période estivale dans les systèmes aquatiques végétalisés, elle est également active en période non estivale dans les zones à sédiments (même non végétalisées). Ceci témoigne du rôle interactif des différents compartiments des écosystèmes aquatiques. La partie amont du cours d'eau forestier de Rhinau fortement végétalisé et comportant une importante phase sédimentaire présente un processus d'amélioration de l'eau, supérieur à celui du Schollengiessen (faiblement végétalisé et essentiellement graveleux).

4) Les tronçons amont du vieuxRhin (de trophie moyenne) présentent une capacité épuratrice sensiblement comparable à celle des tronçons aval, plus contaminés. Ces systèmes aquatiques modérément végétalisés présentent une faible vitesse d'écoulement, et se comportent comme de vastes bras latéraux aux eaux calmes. A ce titre, ils jouent en tant que "nids à plancton" un rôle considérable dans l'assainissement des eaux.

II apparaît donc schématiquement, comme cela avait déjà été souligné en 1969 par Carbiener, que les capa- cités d'auto-épuration et de réduction de la charge eutrophisante d'un système aquatique lotique sont d'autant plus grandes que ce systeme se rapproche de son état naturel (alternance de fosses, de seuils...).

Afin de contribuer à améliorer, ne serait-ce que temporairement, les eaux du Rhin canalisé, il serait souhaitable de multiplier la remise en eaux ou la "revitalisation" de cours d'eau latéraux forestiers. Ceux-ci, devront être équipés d'un système permettant de suspendre momentanément l'alimentation en eau, ce qui permettra de les préserver des contaminations accidentelles qui affectent le Rhin (déversement accidentel de produits toxiques par exemple). Ces cours d'eau pourront alors jouer le rôle de biotopes refuges et de réservoir génétique, susceptibles d'accélérer les processus de restauration du fleuve.

Cette revitalisation de bras latéraux devra être soigneusement étudiée afin de ne pas affecter des cours d'eau phréatiques de très bonne qualité qui présentent actuellement une végétation typique de milieux oligotrophes, sous peine de les condamner à une banalisation de la flore et de la faune aquatique.

\section{BIBLIOGRAPHIE}

Anvieu J.C., 1980. Réaction des phosphates minéraux en milieu calcaire; conséquences sur l'état et la solubilité du phosphore. Sci. de l'eau, 3: 179190. 
Barbe J., 1981. Développement du phytoplancton dans la rivière Doubs: ses causes et ses relations avec la qualité physico-chimique de l'eau. Ann. Scient. de l'Univ. de Franche-Comté. $4^{e}$ série, fasc. 2 : 33-38.

Blake G., 1989. Rôle des végétaux aquatiques vis-à-vis du phosphore dans les écosystèmes et les systèmes d'épuration. Association Internationale des Entretiens Ecologiques. Colloque sur le Phosphore, ses dérivés et leur comportement dans le milieu naturel 22-24 Nov 1988, cahier n² 28 T1, 119132.

Bole J.B., Allan J.R., 1978. Uptake of phosphorus from sediment by aquatic plants, Myriophyllum spicatum and $\mathrm{Hy}$ drilla verticillata. War. Research, 12 : 353-358.

BRGM-SGAL, 1978. Etude de l'invasion des eaux rhénanes dans la nappe phréatique d'Alsace en aval de Fessenheim. Rapport BRGM-SGAL 0133, Strasbourg, Ed. SRAE : 29 p.

Carbiener R., 1969. Aperçu sur quelques effets de la pollution des eaux douces de la zone tempérée sur les biocénoses aquatiques. Bull. de la sect. de Géographie, LXXX, Bibl. Nat. Paris: 45-132.

Carbiener R., 1983. Le Grand Ried Central d'Alsace: écologie et évolution d'une zone humide d'origine fluviale rhénane. Bull. Ecol., 14(4): 249-277.

Carbiener R., 1990. Rapport au Secrétaire d'Etat auprès du Premier Ministre chargé de l'Environnement sur les compositions lessivielles avec ou sans phosphates et protection des milieux aquatiques: $182 \mathrm{p}$.

Carbiener R., Ortscheit A., 1987. Wasserpflanzengesellschaften als Hilfe zur Qualitätsüberwachung eines des grossten Grundwasservorkommens Europas (Oberrheinebene). Proceed. Inter. Symp. IAVS Tokyo-Yokohama 1984, vegetation Ecology and Creation of new Environments, Miyawaki et al., : 284-312.
Carbiener R., Tremolières M., Mercier J.L., Ortscheit A., 1990. Aquatic macrophytes communities as bioindicators of eutrophication in calcareous oligosaprobe stream waters (Upper Rhine plain, Alsace). Vegetation, 86: 71-88.

Carpenter S.R., Adams S.A., 1977. The macrophyte tissue nutrient pool of a hardwater eutrophic lake: implication for macrophyte harvesting. Aquat. Bot., 3: 239-255.

Chen R.L., Barko J.W., 1988. Effects of Freshwater macrophytes on sediment chemistry. Journal of Freshwater Ecology, 4(3): 279-289.

Didenot D., Hennequin M., Martin G., 1987. Chimie analytique des especes phosphorées. Point sur l'épuration et le traitement des effluents (eau air), vol. 3: Phosphore. Ed. Lavoisier Tec et Doc: 31-63.

Eglin I., Robach F., 1992. Typologie et végétation de l'hydrosysteme rhénan dans le secteur central de la plaine d'Alsace: interprétation et fonctionnement écologique. Doct. ULP Strasbourg: $342 \mathrm{p}$

Fogg G.E., 1973. Phosphorus in primary aquatic plants, Wat. Research, 7: 7791.

Kramer J., Herbes S., Allen H., 1972. Phosphorus: analysis of water, biomass and sediment. Nutrient in natural waters. New-York. Wiley-International Publication: $51-100$.

Ombredanne D., Fenin P., Morvan J., Martin G., 1982. Etude en laboratoire de l'influence des sédiments sur l'évolution des formes azotées de l'eau dans un systeme biotique fonctionnant en dynamique. Journ. Français. D'Hydrol. 13(3): 237-253.

Pilleboue-Baptendier E., 1987. Origines, bilans et mécanismes de transfert du phosphore et de l'azote d'un bassin versant vers un lac. Thèse de Doct. Univ. Paris 6. Géol. Appliquée; Option Sc. de l'eau: $251 \mathrm{p}$. 
Radoux M., 1986. Epuration de rejets domestiques par marais reconstitué, station expérimentale de Viville. Contrat CEE-FUL Environnement 634B: 113 p.

Radoux M., Kemp D., 1988. Epuration comparée des eaux usées domestiques par trois plantations hélophytiques et par un lagunage à microphytes sous un même climat tempéré. Act. Oecologica, 9: 25-38.

Robach F., Eglin I., 1988. Contribution à l'étude de l'auto-épuration d'eaux polluées d'origine rhénane et répartition de la végétation aquatique dans le cadre hydrologique complexe de l'île de Rhinau. Mémoire de DEA. ULP Strasbourg I: $90 \mathrm{p}$.

Robach F., Eglin I., Carbiener R., 1991.
Hydrosystème rhénan : évolution parallèle de la végétation aquatique et de la qualité de l'eau (Rhinau). Bull. Ecol., 22: 227-241.

Sanchez-Perez J.M., 1987. Variabilité spatiale de la matière organique en moyenne montagne tempérée: ses relations avec les caractéristiques hydriques des sols. Discrétisation de l'espace en zones homogènes (BV de la Fecht, Vosges moyennes, France). DEA, ULP Strasbourg I: $79 \mathrm{p}$.

Sanchez-Perrez J.M., Tremolières M., Carbiener R., 1991. Une station d'èpuration naturelle des phosphates et nitrates apportés par les eaux de débordement du Rhin: la forêt alluviale à frêne et orme. C.R. Acad. Sci. Paris, 312 (III) : 395-402.

Adapté d'une communication présentée au colloque international de Besançon (16-19 novembre 1992) "Les acquis de la limnologie et la gestion des systèmes aquatiques continentaux". 